\title{
PERIDYNAMIC MODELING OF GRANULAR FRACTURE IN POLYCRYSTALLINE MATERIALS
}

\author{
Dennj De Meo ${ }^{1}$, Ning Zhu ${ }^{1}$, Erkan Oterkus ${ }^{1, *}$ \\ ${ }^{1}$ Department of Naval Architecture, Ocean and Marine Engineering, University of Strathclyde, Glasgow, \\ Lanarkshire, G4 OLZ, UK
}

\begin{abstract}
A new peridynamic formulation is developed for cubic polycrystalline materials. The new approach can be a good alternative to traditional techniques such as finite element method and boundary element method. The formulation is validated by considering a polycrystal subjected to tension loading condition and comparing the displacement field obtained from both peridynamics and finite element method. Both static and dynamic loading conditions for initially damaged and undamaged structures are considered and the results of plane stress and plane strain configurations are compared. Finally, the effect of grain boundary strength, grain size, fracture toughness and grain orientation on time-to-failure, crack speed, fracture behaviour and fracture morphology are investigated and the expected transgranular and intergranular failure modes are successfully captured. To the best of the authors' knowledge, this is the first time that a peridynamic material model for cubic crystals is given in detail.
\end{abstract}

Keywords: transgranular fracture; polycrystalline materials; peridynamics; dynamic fracture; crack branching; intergranular fracture

\section{INTRODUCTION}

Polycrystalline materials, such as common metals, rocks and many ceramics, are solids constituted by crystals (or grains) that can have different shape, size and random orientation and are attached to each other through their grain boundaries. As argued in [1], polycrystalline materials are among the most common materials used in practical engineering applications. Hence, in order to build safe structures without relying on excessive overdesign, it is necessary to fully understand the fracture behaviour of these materials. However, owing to the fact that the fracturing of polycrystalline materials is often influenced by the characteristics of its microstructure (e.g. grain size, differences in physical properties between grain and its boundaries, crystallographic orientation and the presence of flaws of different size, shape and orientation), the task is often challenging.

Several experimental approaches are available to study the fracture behaviour of this class of materials [2-4]. Unfortunately, despite the resulting valuable information provided by experimental approaches, these techniques are not always viable due to the necessity of

\footnotetext{
${ }^{1}$ Corresponding author
} 
expensive equipment and complex and time consuming procedures for material preparation and analysis [1]. Therefore, computational approaches represent a precious complement for understanding the fracturing of polycrystalline materials. In this regard, several computational techniques have been employed to date and many of them are based on the cohesive zone model (CZM) implemented within the framework of the finite element method (FEM) [5-8]. On the other hand, Sukumar et al. [9] investigated the intergranular and transgranular fracturing of brittle polycrystalline materials by using the extended finite element method (XFEM), whereas the boundary element method (BEM) coupled with CZM was used for the modelling of fracture in alumina [10] and $\mathrm{SiC}$ [11] and for the polycrystalline modelling of fcc nickel [12], SiC [1] and various cubic polycrystal systems [13]. As argued by [10], despite the valuable results obtained by the latest numerical studies, the mathematical modelling of the transition from microscopic defects to macroscopic cracks is not entirely understood at present and, as a result, the current design codes and standards prescribe the use of safety factors to compensate this lack of understanding and predicting capability [14]. As pointed out by [15], one of the main reasons for this difficulty is given by the mathematical formulation of classical continuum mechanics (CCM) (i.e. the theory behind several computational techniques used for structural analysis), which employs partial differential equations and assumes that the body remains continuous as it deforms. Moreover, as argued by [10], continuum damage mechanics is not suitable for predicting the initiation and propagation of micro-cracks at small scales, especially in the case of polycrystalline materials [11, 14]. Other reasons for the difficulty of modelling the fracturing of polycrystalline materials by using computational techniques based on CCM are specific to the particular numerical approach employed. For instance, as mentioned in [16], the solution obtained from the XFEM lacks accuracy in proximity of the crack tip and as a consequence, according to [15], the XFEM is not suitable for the prediction of complex fracture scenarios where multiple cracks initiate, grow and interact. Concerning the numerical techniques based on the coupling of FEM with CZM, it is well-known that the FEM leads to crack growth predictions that are dependent on the mesh size, no matter which numerical crack growth strategy is used [17]. Moreover, as pointed out in [15], the stiffness of FEM models is also dependent on the mesh size, owing to the fact that a decrement of mesh size often corresponds to an increment of the number of cohesive elements present in the model. Lastly, the nucleation of micro-cracks leads to modifications of the elastic stiffness of FEM models, which eventually results in ill-posed problems [10]. 
With the aim of overcoming some of the above mentioned issues concerning the prediction of material failure shown by numerical techniques based on CCM, peridynamics (PD), a nonlocal generalisation of CCM based on integral equations rather than partial differential equations, was introduced by Silling in [18]. An extensive review of PD studies is given in [15]. Askari et al. [19] performed PD simulations of granular fracture in a silicon polycrystal (cubic system); they investigated the effect of the grain boundary strength on the fracture mechanisms. Recently, Ghajari et al. [20] applied PD for modeling the dynamic fracture response of alumina (hexagonal system).

In this study, a new PD model is proposed for cubic crystals and the parameters of the PD material model are obtained by equating the PD and the CCM strain energy density expressions of a body subjected to simple loading conditions. The proposed model is validated by considering a polycrystal subjected to tension loading and comparing the displacement field obtained from both PD and FEM. Both static and dynamic loading conditions for initially damaged and initially undamaged structures are considered and the results of plane stress and plane strain configurations are compared. Finally, the effect of the grain boundary strength, grain size, fracture toughness and grain orientation on time-tofailure, crack speed, fracture morphology and fracture behaviour of the material are investigated and the expected transgranular and intergranular failure modes are successfully captured. To the best of authors' knowledge, this is the first time that a PD material model for cubic crystals is given in detail.

\section{PERIDYNAMIC THEORY}

As mentioned earlier, the equation of motion of a material point in CCM can be expressed in the form of a partial differential equation which can be written as

$$
\rho(\mathbf{x}) \ddot{\mathbf{u}}(\mathbf{x}, t)=\nabla \cdot \sigma+\mathbf{b}(\mathbf{x}, t)
$$

where $\rho(\mathbf{x})$ and $\ddot{\mathbf{u}}(\mathbf{x}, t)$ denote the density and acceleration of the material point $\mathbf{x}$ at time $t$, respectively. In Eq. (1), $\sigma$ represents the stress tensor and the term $\mathbf{b}(\mathbf{x}, t)$ is the body force acting on material point $\mathbf{x}$ at time $t$. The operator $\nabla \cdot$ represents a divergence operator. Although Eq. (1) has been successfully applied to many different problems of solid mechanics, the derivatives in space are not defined if there is any discontinuity in the material. This problem can be overcome by replacing the divergence term in Eq. (1) with an integration as [18] 


$$
\rho(\mathbf{x}) \ddot{\mathbf{u}}(\mathbf{x}, t)=\int_{H_{\mathbf{x}}} \mathbf{f}\left(\mathbf{u}\left(\mathbf{x}^{\prime}, t\right)-\mathbf{u}(\mathbf{x}, t), \mathbf{x}^{\prime}-\mathbf{x}\right) \mathrm{dV}_{\mathbf{x}^{\prime}}+\mathbf{b}(\mathbf{x}, t)
$$

where $\mathbf{u}(\mathbf{x}, t)$ denotes the displacement of the material point $\mathbf{x}$ at time $t$ and $\mathbf{f}\left(\mathbf{u}\left(\mathbf{x}^{\prime}, t\right)-\mathbf{u}(\mathbf{x}, t), \mathbf{x}^{\prime}-\mathbf{x}\right)$ represents the PD force between material points $\mathbf{x}$ and $\mathbf{x}^{\prime}$ (also called mechanical response function), respectively. According to this new formulation, a material point can interact with other material points not only within its nearest neighbourhood, but also with material points in a larger neighbourhood. Since the strength of the interaction between material points decreases as the distance between them increases, an influence domain, named horizon, $H_{\mathrm{x}}$, can be defined for each material point as shown in Fig. 1. Therefore, the material point $\mathbf{x}$ can only interact with material points within this domain. The radius of the horizon, $\delta$, is chosen depending on the nature of the problem in such a way that the model is able to fairly represent the physical mechanisms of interest [21].

In the case of an elastic material, the peridynamic force between material points $\mathbf{x}$ and $\mathbf{x}^{\prime}$, can be expressed as:

$$
\mathbf{f}=c s \frac{\mathbf{y}^{\prime}-\mathbf{y}}{\left|\mathbf{y}^{\prime}-\mathbf{y}\right|}
$$

where $\mathbf{y}$ represents the location of the material point $\mathbf{x}$ in the deformed configuration, i.e. $\mathbf{y}=\mathbf{x}+\mathbf{u}$ and $c$ is the bond constant which can be related to material constants of CCM. In Eq. (3), the stretch parameter $s$ is defined as:

$$
s=\frac{\left|\mathbf{y}^{\prime}-\mathbf{y}\right|-\left|\mathbf{x}^{\prime}-\mathbf{x}\right|}{\left|\mathbf{x}^{\prime}-\mathbf{x}\right|}
$$

In the case of brittle material behaviour, the peridynamic force and the stretch relationship are shown in Fig. 2.

The parameter $s_{0}$, in Fig. 2, is called critical stretch and if the stretch of a peridynamic bond exceeds this critical value, then the peridynamic interaction (bond) is broken. Hence, the peridynamic force between the two material points reduces to zero. 


\section{PERIDYNAMIC MICRO-MECHANICAL MODEL FOR CUBIC CRYSTALS}

In this study, a microscopic material model is used to represent the behaviour of a polycrystalline structure with random texture. For this purpose, a new peridynamic material model is developed to express the deformation response of each crystal. For simplicity, the bond-based PD framework has been chosen over the more advanced state-based version of PD, which allows to overcome the restriction to $1 / 3$ (2D) and $1 / 4$ (3D) of the value of the Poisson's ratio. However, for the materials considered in this study, the bond-based PD is suitable. The polycrystalline structure is generated by using the Voronoi tessellation method (Fig. 3).

The micro-mechanical PD model for cubic crystals is constituted by the following two types of PD bonds (Fig. 4):

- Type-1 bonds (dashed green lines) - exists in all directions (i.e. $\theta=0-2 \pi$ )

- Type-2 bonds (solid red lines) - exists only for the following directions:

$$
\theta=\frac{\pi}{4}, \frac{3}{4} \pi, \frac{5}{4} \pi, \frac{7}{4} \pi
$$

Note that the angle $\theta$ is defined with respect to the orientation of the crystal. In the special case shown in Fig. 5, the crystal orientation $\gamma$ equals $\pi / 4$ and it is always measured with respect to the $\mathrm{x}$-axis, while, in general, an algorithm is used to assign a random orientation $\gamma$ to the grain. As a result of this procedure, when a polycrystalline system of random texture is represented by this model, type-2 bonds will exist in many different directions according to the random orientations of the crystals.

The bond constants for type- 1 and type-2 PD bonds can be expressed in terms of the material constants of a cubic crystal, $C_{i j}$, by following a procedure similar to that explained in [22]. In the case of plane stress condition, the bond constants can be expressed as:

$$
c_{T 1}=\frac{12\left(c_{11}^{2}-c_{11} c_{12}\right)}{\pi h \delta^{3} c_{11}} \quad, \quad c_{T 2}=\frac{4\left(3 c_{11} c_{12}-2 c_{12}{ }^{2}-c_{11}^{2}\right)}{\left(\beta_{A}+\beta_{B}\right) c_{11}}
$$

where $h$ is the thickness of the structure. The quantities $\beta_{A}$ and $\beta_{B}$ can be expressed as

$$
\beta_{A}=\sum_{j=1}^{q_{A}} \xi_{i j} V_{j} \quad, \quad \beta_{B}=\sum_{j=1}^{q_{B}} \xi_{i j} V_{j}
$$


where subscript $A$ is associated with directions $\theta=\frac{\pi}{4}, \frac{5}{4} \pi$, whilst subscript $B$ is associated with directions $\theta=\frac{3}{4} \pi, \frac{7}{4} \pi$.

In Eq. (6), $i$ and $j$ refer to a generic particle and its neighbour, respectively, $V_{j}$ denotes the volume of particle $j, \xi_{i j}$ is the initial length of the bond between particles $i$ and $j$, and $q_{A}$ and $q_{B}$ represent the number of PD bonds along the directions associated with $A$ and $B$, respectively. Similarly, in the case of plane strain condition, the bond constants can be expressed as:

$$
c_{T 1}=\frac{12\left(c_{11}-c_{12}\right)}{\pi h \delta^{3}} \quad, \quad c_{T 2}=\frac{4\left(3 c_{12}-c_{11}\right)}{\left(\beta_{A}+\beta_{B}\right)}
$$

The detailed derivation of type- 1 and type-2 PD bond constants for both plane stress and plane strain configuration is given in Appendix A. The model was fully validated by using the FEM's deformation field as a reference and a good agreement between the two approaches was observed as shown in Section 5.1 and 5.2. The critical stretch parameter for PD bonds was obtained based on the expression given in [15]:

$$
s_{0}=\sqrt{\frac{4 \pi G_{c}}{9 E \delta}}
$$

where $E$ is the Young's modulus. In case of linear elastic material, the critical energy release rate $G_{c}$ can be obtained from the fracture toughness, $K_{I c}$, for plane stress and plane strain configurations, respectively, as follows [17]:

$$
\begin{gathered}
G_{c}=\frac{K_{I c}^{2}}{E} \\
G_{c}=\frac{K_{I c}^{2}}{E}\left(1-v^{2}\right)
\end{gathered}
$$

In order to investigate various fracture modes typical of polycrystalline materials, a grain boundary coefficient (GBC) is defined as follows:

$$
G B C=\frac{s_{0 G B}}{s_{0 G I}}
$$


where $s_{0 G B}$ and $s_{0 G I}$ represent the critical stretch of the PD bonds that cross the grain boundaries of the material and the critical stretch of the PD bonds that are fully included within the grains, respectively (GB stands for grain boundary, while GI stands for grain interior).

\section{MATERIAL DATA}

Two materials are employed in this study. Iron crystals are considered for the static analyses (Section 5.1 and Section 5.2), while AISI 4340 steel is considered for the dynamic analyses (Section 5.3, Section 5.4 and Section 5.5). The chemical composition of AISI 4340 steel is summarised in Table 1 [23]:

The fracture toughness of AISI 4340 steel is considered to be $K_{I c}=58.4 \mathrm{MPa} \sqrt{\mathrm{m}}$ [24]. The crystals are assumed to have $\alpha$-ferrite structure with bcc lattice. As described in [25], the local stiffness matrix of each individual crystal can be written as:

$$
[C]=\left[\begin{array}{cccccc}
c_{11} & c_{12} & c_{12} & 0 & 0 & 0 \\
c_{12} & c_{11} & c_{12} & 0 & 0 & 0 \\
c_{12} & c_{12} & c_{11} & 0 & 0 & 0 \\
0 & 0 & 0 & c_{44} & 0 & 0 \\
0 & 0 & 0 & 0 & c_{44} & 0 \\
0 & 0 & 0 & 0 & 0 & c_{44}
\end{array}\right]
$$

In order to take into account the polycrystalline nature of the material, the values of the elastic moduli $C_{i j}$ are found by applying an axial tension to a microscopic AISI 4340 steel specimen and then fitting experimental and numerical results as described in [26]. The resulting microscopic material properties are:

$$
c_{11}=208.9 \quad \mathrm{GPa} \quad c_{12}=126.4 \quad \mathrm{GPa} \quad c_{44}=97.7 \quad \mathrm{GPa}
$$

Concerning iron crystal, the considered microscopic material properties are [25]:

$$
c_{11}=231.4 \quad \mathrm{GPa} \quad c_{12}=134.7 \quad \mathrm{GPa} \quad c_{44}=116.4 \quad \mathrm{GPa}
$$

\section{NUMERICAL RESULTS AND DISCUSSION}

In this section, the results obtained from static and dynamic PD analyses are presented, and comparisons with FEM results are also provided. Concerning the static analyses, firstly 
(Section 5.1), a single cubic crystal is considered and the PD and FEM displacement fields are compared. Secondly (Section 5.2), a cubic polycrystal constituted by 100 grains is analysed and, again, the PD and FEM displacement fields are compared. Concerning the dynamic analyses, first the influence of the horizon and peridynamic discretization on results is evaluated (Section 5.3.1). Secondly (Section 5.3.2), the effect of the grain size and grain orientation on the time-to-failure and fracture behaviour is investigated. Thirdly (Section 5.3.3), the impact of GBC on the crack speed is examined. Finally, a comparison of fracture morphology is provided for different values of $K_{I C}$ (Section 5.3.4) and for plane stress/strain conditions (Section 5.3.5).

\subsection{Static analysis of a cubic crystal}

The cubic crystal considered in this study has a length of $15.24 \mu \mathrm{m}$ and a width of $7.62 \mu \mathrm{m}$ (Fig. 5). The number of particles along the horizontal and vertical directions is 240 and 120 respectively. The left edge of the crystal is fully fixed, while the right edge is subjected to a horizontal loading of $P=156 \mathrm{MPa}$.

Three layers of virtual particles are placed along the right edge of the plate to impose the tension loading boundary condition. Three additional layers of virtual particles are also placed along the left edge of the plate with the aim to constrain the model. The values of grid spacing and horizon radius are $\Delta x=6.35 \cdot 10^{-2} \mu \mathrm{m}$ and $\delta=19.14 \cdot 10^{-2} \mu \mathrm{m}$, respectively.

Fig. 6 and Fig. 7 show the comparison between the results obtained with FEM and PD analyses concerning the displacement field of the cubic crystal in plane stress configuration with orientation $0^{\circ}$ (i.e. the orientation of the crystal coincides with the $x$-direction) and orientation $45^{\circ}$, respectively.

Fig. 8 and Fig. 9 show the comparison between the results obtained with FEM and PD concerning the displacement field of the cubic crystal in plane strain configuration with orientation $0^{\circ}$ (i.e. the orientation of the crystal coincides with the $\mathrm{x}$-direction) and orientation $45^{\circ}$, respectively.

The same simulations have been carried out considering also other static loading conditions; in all cases, a good agreement was found between FEM and PD results. Therefore, in conclusion, the micro-mechanical PD model for cubic crystals presented in this paper agrees well with the FEM for plane stress configuration, for plane strain configuration, for different grain orientation and for different static loading conditions.

\subsection{Static analysis of a cubic polycrystal}


The cubic polycrystal considered in this section is constituted by 100 randomly oriented grains and has a length of $5 \mathrm{~mm}$ and a width of $2.5 \mathrm{~mm}$ (Fig. 10). Similar to the previous analysis (Section 5.1), the number of particles along the horizontal and vertical direction is 240 and 120 respectively. The left edge of the polycrystal is fully fixed while the right edge is subjected to a horizontal loading of $P=150 \mathrm{MPa}$. Three layers of virtual particles are placed along the right edge of the plate to impose the tension loading boundary condition. Three additional layers of virtual particles are also placed along the left edge of the plate with the aim to constrain the model. The values of grid spacing and horizon radius are $\Delta x=2.083 \cdot 10^{-5} \mathrm{~m}$ and $\delta=6.281 \cdot 10^{-5} \mathrm{~m}$, respectively.

As shown in Fig. 11 and Fig.12, a good agreement between FEM and PD was found concerning the final displacement field of the cubic polycrystal in plane stress (Fig.11) and plane strain configurations (Fig.12).

\subsection{Dynamic analysis of AISI 4340 polycrystals}

\subsubsection{Selection of the horizon size}

The aim of this analysis is to investigate the effect of the horizon size and peridynamic discretization on the fracture pattern predicted by our PD model. For this purpose, an AISI 4340 polycrystal in plane strain condition with dimensions of $5 \mathrm{~mm}$ by $5 \mathrm{~mm}$ is considered as shown in Fig. 13. Both left and right edges of the polycrystal are subjected to a horizontal velocity boundary condition of $V=25 \mathrm{~m} / \mathrm{s}$. Three layers of virtual particles are placed along the left and right edges of the plate to impose the velocity boundary condition. Moreover, in order to ensure the external load to be transferred properly to the internal part of the plate, a no-fail zone is imposed on the virtual particles and their neighbours. The length of the two initial cracks at the bottom and top edges of the polycrystal is $0.4 \mathrm{~mm}$ (see Fig. 13). An explicit central difference scheme is used for the time integration with a time step size of $d t=2 \mathrm{~ns}$. The total simulation time is $2.4 \mu$ s corresponding to 1200 time steps. This study considers three different GBC values (0.5, 1.0 and 2.0), three different values of average grain size $(333 \mu \mathrm{m}, 416 \mu \mathrm{m}$ and $714 \mu \mathrm{m})$ and five different values of horizon size, $\delta(202.7$ $\mu \mathrm{m}, 100 \mu \mathrm{m}, 50 \mu \mathrm{m}, 37.5 \mu \mathrm{m}$ and $30 \mu \mathrm{m}$ ) for a total of 45 simulations (see Figs. 14-16). The horizon size is calculated as $\delta=3.015 \cdot \Delta x$. Therefore, its value is controlled indirectly by changing the PD discretization, i.e. 74 × 74 particles, $150 \times 150$ particles, $300 \times 300$ particles, $400 \times 400$ particles and $500 \times 500$ particles, respectively. 
As depicted in Fig. 14, for the polycrystal with average grain size of $333 \mu \mathrm{m}$, a horizon size of $202.7 \mu \mathrm{m}$ (top red square with arrow) is sufficient to reproduce the main features of the fracture pattern. Indeed, in all five simulations, the major cracks propagate from the initial notch and the fragmentation of the central part of the plate is similar despite the poor resolution of the results obtained with higher horizon sizes and lower number of particles. In other words, the horizon size and the PD discretization affect the resolution of the results, but not the overall fracture pattern. This is not true for higher values of average grain size. For the simulations with average grain size of $416 \mu \mathrm{m}$ and $714 \mu \mathrm{m}$, the convergent horizon values are $100 \mu \mathrm{m}$ (middle red square with arrow) and $50 \mu \mathrm{m}$ (bottom red square with arrow), respectively. This can be explained by noting that if the dimensions of the plate are kept constant as in this case, the smaller the grain size the higher the possibility that a grain boundary exists in proximity of the initial crack tip. If the grain boundary is weak with respect to the bulk of the crystal (like in this case since $\mathrm{GBC}=0.5$ ), the initial notch is further encouraged to propagate and form a major crack despite the coarse mesh.

The simulations that considered higher horizon values and lower number of particles than the convergent ones failed to capture the right location of the major cracks. Therefore, when $\mathrm{GBC}=0.5$, the value of the convergent horizon size is dependent on the grain size. As it can be seen in Figs. 15-16, this is not true for GBC $=1.0$ and $\mathrm{GBC}=2.0$. In these cases, the grain boundaries do not correspond to the part of weak points anymore and, as a result, in both cases, the grain size does not influence the value of the convergent horizon (red squares with arrow), which can be considered to be $50 \mu \mathrm{m}$ ( $300 \times 300$ particles) for all values of grain size considered. Moreover, the fracture patterns in Fig. 15-16 are very similar and the failure mode is transgranular. This is in contrast with the results for GBC $=0.5$ (Fig. 14), where the fracture patterns are different with respect to Fig. 15-16 and the failure mode is intergranular. A more detailed analysis of the effect of GBC is provided in Section 5.3.3 and Section 5.3.4.

\subsubsection{Grain size effect on time-to-failure and fracture behaviour}

The aim of this section is to investigate the effect of the grain size on the time-to-failure, i.e. the time-lapse between the application of the load and the propagation of the initial notch, and fracture behaviour. For this purpose, a double-edge notched plate, shown in Fig. 13, with $300 \times 300$ particles and GBC $=0.5$ is considered. Same boundary conditions, time step size, time integration scheme and values of grain size are used as in Section 5.3.1. For each of the three values of grain size, ten simulations are performed (each one with random grain 
orientation) for a total of twenty data points for each grain size, i.e. two data points for each simulation since the plate has two notches. This is done to distinguish our conclusions on the effect of the grain size from the effect of the grain orientation.

As already mentioned in Section 5.3.1, if the dimensions of the plate remain the same, the smaller the grain size the higher the volume fraction of grain boundaries and the higher the probability that a grain boundary exists in proximity of the initial crack tip. Therefore, if we define the occurrence as the event in which the initial notch propagates to form a major crack (see Fig. 17), it is expected that the smaller the grain size the higher the number of occurrences.

However, PD results contradict to this expectation. As shown in Fig. 18, the percentage of occurrences (i.e. the number of occurrences divided by 20) is approximately the same for all values of grain size considered in this study. More precisely, it has a slight tendency to increase as the grain size increases. The video of the simulations have also helped in shedding some light on this unexpected result. According to the authors, there are two competing mechanisms in place. The first one is the mechanism mentioned earlier and it was expected before running the simulations, i.e the smaller the grain size the higher the probability that a grain boundary exists in proximity of the initial crack tip, which encourages the propagation of the initial notch (see bottom notch in Fig. 17) and raises the number of occurrences. The second mechanism can be described as follows: the smaller the grain size, the higher the volume fraction of grain boundaries, the higher the number of weak points within the material and, as a result, the higher the probability of new cracks nucleating, propagating and protecting/shielding the initial notch from the external load. This second mechanism can be visualized in Fig. 17, where the top notch is clearly shielded by the nucleation and propagation of two adjacent cracks. In contrast, no crack nucleates in proximity of the bottom notch. Hence, the notch is not shielded, and propagates and leads to the formation of a major crack.

This finding reminds us a particular toughening mechanism called microcrack cloud. As described by Lawn in [27], this is a mechanism induced by the occurrence of microcraks in proximity of the crack tip (see Fig. 19), which act as stress relievers and impose a dilatant closure field on the crack. This phenomenon is an open research topic in the field of fracture of polycrystalline materials and quantitative prediction techniques are not well-established due to the extremely complex nature of the phenomenon [28, 29]. As argued in [30], 
experimental techniques such as TEM are affected by detectability limitations, while theoretical models cannot fully clarify the mechanical degradation produced by the formation and interaction of microcraks. Analytical, semi-analytical and numerical studies are available (e.g. [31-33]), but they always require artificial conditions [33]. As mentioned in [32], they often assume fixed and already nucleated microcrack configurations and do not consider the polycrystalline nature of the material. We quote from page 216 in [27]: ...The issue of microcrack toughening raises two fundamental questions: what are the condition that a microcrack cloud should initiate in the field of a primary crack?; given that these conditions are met, what is the toughness increment?... Returning to our PD results, it would be interesting to understand why only the top notch in Fig. 17 benefits from the shielding effect. According to the authors, several factors could be responsible for this situation, e.g. the grain orientation, the value of GBC, the grain size, the magnitude of the load, the type of load and the morphology of the crystals. For this study, we limit ourselves to report this finding and a detailed investigation of these effects will be considered as future work.

The results concerning the effect of the grain size on the time-to-failure is presented in Fig. 20. As already mentioned, ten simulations are performed (each simulation has a random grain orientation) for each grain size. Moreover, the damage index in proximity of the crack tip is monitored and 20 data points (i.e. the time-to-failure) for each grain size are collected, for a total of 60 data points. Fig. 20 is constructed by considering only the time-to-failure of occurrences, i.e. the value of the time-to-failure is only determined if the initial notch propagates and forms a major crack such as the bottom notch in Fig. 17. Since the number of occurrences depends on the grain size, the curves in Fig. 20 are constituted by a different number of data points. By observing Fig. 20, it can be concluded that the average time-tofailure is influenced by the grain size. More precisely, the higher the grain size the higher the average time-to-failure. The first mechanism mentioned above, i.e. the smaller the grain size the higher the probability that a grain boundary happens to be in proximity of the initial crack tip, is probably responsible for this effect. In contrast, the second mechanism, i.e. microcrack shielding, is excluded here since only the time-to-failure data of occurrences is considered. Another important point is the amplitude of fluctuation of the curves in Fig. 20. It is observed that the lower the grain size the lower the amplitude. This behaviour is reasonable since a smaller grain size generally means a more homogeneous polycrystal, which is also supported by the findings reported in [11], where the mechanical behaviour of a $\mathrm{SiC}$ polycrystal in tension is analysed by using a boundary cohesive grain element formulation. The reason for 
the fluctuations in Fig. 20 can be found by looking at the damage pattern of the polycrystal at the end of the simulation, i.e. time $=2.4 \mu$ s as in Section 5.3.1. For example, let's consider the lowest point of the blue curve. As can be seen in Fig. 21, the crack tip of the initial notch is located in proximity of one of the grain boundaries of the polycrystal. Hence, the value of the time-to-failure is lower than the average time-to-failure. In other words, the initial notch starts to propagate earlier. In contrast, if the peak value of the blue curve is considered and the final fracture pattern of the polycrystal shown in Fig. 22 is analyzed, it can be seen that the initial notch is further from the nearest grain boundary, leading to a delay in the onset of the propagation of the initial notch.

\subsubsection{GBC effect on crack propagation speed}

The scope of this analysis is to investigate the effect of GBC on the crack propagation speed. For this purpose, same configuration is considered as in Section 5.3.2 with the following three modifications: 1) the bottom notch is removed from the model, 2) the length of the initial notch is increased from $0.4 \mathrm{~mm}$ to $1.4 \mathrm{~mm}$ and 3) the velocity boundary condition is substituted by an opening load applied instantaneously at the flanks of the initial notch as in the experimental study reported in [34] and in the numerical study reported in [35] and kept constant throughout the simulation. The reason for these modifications is that we want to reproduce a situation where a single crack propagates from the initial notch without any new crack nucleating in other parts of the plate, which would make more difficult to track the crack tip and the calculation of the crack speed is more ambiguous. Four different simulations are carried out by considering the following values of GBC: 0.7, 1, 5 and 10. The value of the applied loading is $1500 \mathrm{MPa}$, the total simulation time is $3.2 \mu \mathrm{s}$, the average grain size is $333 \mu \mathrm{m}$ and the grain orientation is always the same in all four simulations. An ad-hoc algorithm is used to track the position of the crack tip, which is identified by a damage index value of 0.35 . In the case of crack branching as seen in the first picture in Fig. 24, the longest branch is followed and considered for the calculation of the crack speed.

As can be seen in Fig. 23, the lower the GBC the lower the time-to-failure, i.e. the initial notch starts to propagate earlier, which is in agreement with our expectations. However, the time-to-failure of the simulations with $\mathrm{GBC}=5$ and $\mathrm{GBC}=10$ is about the same, meaning that the effect above mentioned may be active only in a limited range of GBC; further investigation concerning this range will be provided in a subsequent paper. After fracture initiation, the crack speed increases very quickly in all four cases and, after having reached a 
peak value, it decreases sharply. The overall trend of the crack speed is in good qualitative agreement with the numerical results reported in [35-37], where PD is used to predict the failure of a single-edge notched specimen subjected to an impulsive opening load. Another important point is related with the peak value of the crack speed. As shown in Fig. 23, the lower the GBC the greater the peak value of the crack speed, which is logical since the weak grain boundaries are served as a path of preferential failure. The final important point is related with the significant difference in final crack speed between the simulation with GBC $=1$ and the other three simulations (see Fig. 23). When GBC $=0.7$, the crack is further slowed down by branching which allows the energy to be dissipated in multiple directions. The branching behaviour does not occur in the other three simulations. In contrast, for the cases $\mathrm{GBC}=5$ and $\mathrm{GBC}=10$, the crack is further slowed down by the augmented resistance of the grain boundaries, which hinder the crack propagation.

\subsubsection{The effect of $K_{I C}$ on fracture morphology}

The aim of this section is to investigate the effect of $K_{I C}$ on fracture morphology. For this purpose, the same configuration analysed in Section 5.3.3 is considered with the only difference being the average grain size, which changes from $333 \mu \mathrm{m}$ to $416 \mu \mathrm{m}$. Five different simulations are carried out by considering the same polycrystal with $\mathrm{GBC}=1$ and the following values of $\psi: 0.2,0.35,0.5,1$ and 2 where $\psi$ is defined as:

$$
\psi=\frac{K_{I C}^{*}}{K_{I C}}
$$

In Eq. (14), $K_{I C}^{*}$ is the effective value of fracture toughness used for the simulations whereas $K_{I C}$ is the nominal fracture toughness of the material, i.e. $58.4 \mathrm{MPa} \sqrt{\mathrm{m}}$. It is worth noting that, as given in Eqs. 7-9, there is a relationship between the fracture toughness and the critical stretch $s_{0}$. Therefore, as the fracture toughness, $K_{I C}^{*}$, changes, the critical stretch, $s_{0}^{*}$, used in our simulations also changes. As shown in Fig. 25, for the values of $\psi \leq 0.5$, crack branching appears to be significant. Moreover, in this range of values of $\psi$, it is observed that the lower the value of $\psi$ the higher the number of branches. This is in qualitative agreement with the finding reported by Espinosa and Zavattieri in [7], where a similar analysis in carried out for a polycrystalline material subjected to impact load by using FEM and cohesive elements. 


\subsubsection{Plane stress vs. plane strain and intergranular/transgranular fracture mode}

The aims of this section are 1) to understand how the morphology of fracture changes when plane stress condition is considered rather than the plane strain condition and 2) to capture the intergranular/transgranular failure modes when the value of GBC is varied. For this purpose, a $5 \mathrm{~mm}$ x $5 \mathrm{~mm}$ double-edge notched polycrystal is constituted by considering 150 randomly oriented grains. The peridynamic discretization is created with $150 \times 150$ particles. An explicit central difference scheme is used for the time integration with the total simulation time of $4 \mu \mathrm{s}$ and the time step size of $d t=2 \mathrm{~ns}$. Both left and right edges of the polycrystal are subjected to a horizontal velocity boundary condition of $V=50 \mathrm{~m} / \mathrm{s}$. Three layers of virtual particles are placed along the left and right edges of the plate to impose the velocity boundary condition. Moreover, in order to allow the external load to be transferred to the inside of the plate, a no-fail zone is imposed on the virtual particles and their neighbours. The values of grid spacing and horizon's radius are $\Delta x=3.33 \cdot 10^{-5} \mathrm{~m}$ and $\delta=1.005 \cdot 10^{-4} \mathrm{~m}$, respectively. As shown in Fig. 13, the length of the two initial cracks at the bottom and top edge of the polycrystal is $0.4 \mathrm{~mm}$. Three different values of GBC are considered to investigate the intergranular and transgranular fracture modes of the polycrystal.

As given in Eqs. 7-9, the only difference between plane stress and plane strain configurations in our PD model is the value of the critical stretch, which is lower in the case of plane strain configuration. Fig. 26-28 show the dynamic response of the polycrystal in plane stress condition at four different times for $\mathrm{GBC}=0.5, \mathrm{GBC}=1.0$ and $\mathrm{GBC}=2.0$, respectively. In contrast, Fig. 29-31 show the dynamic response of the polycrystal in plane strain condition at four different times for $\mathrm{GBC}=0.5, \mathrm{GBC}=1.0$ and $\mathrm{GBC}=2.0$, respectively.

The time-to-failure and the overall level of final damage of the structures in plane stress configuration and plane strain configuration with different values of GBC are comparable whereas the aspect of damage is different. Indeed, when GBC $=0.5$, the fracture mode appears to be intergranular. On the other hand, when $\mathrm{GBC}=1.0$ and $\mathrm{GBC}=2.0$, the fracture mode is similar and prevalently transgranular, which is in qualitative agreement with [19], where PD is used to study the transition intergranular/transgranular failure mode in polycrystalline materials. Moreover, two major differences can be noticed when comparing the results of plane stress and plane strain configurations. Firstly, the gap between the flanks of the initial notch appears to be wider in case of plane stress configuration. Secondly, a greater level of fragmentation can be noticed in the case of plane strain configuration. Despite 
the fact that the material employed in these simulations is the same, both the two previously mentioned features make the aspect of damage qualitatively more brittle in the case of plane strain configuration since the value of the critical stretch is lower in the case of plane strain configuration, which is in agreement with the findings of Section 5.3.4.

\section{CONCLUSION}

In this paper, a novel peridynamic formulation for cubic crystals has been introduced and all the relevant derivations have been provided. Static analyses have been carried out by considering different grain orientations, different loading conditions and different configurations (plane stress and plane strain). In all cases, a good quantitative agreement has been found between PD and FEM results. Dynamic analyses have also been carried out for different specimen configurations and loading conditions with the aim to investigate the effect of grain size, grain orientation, grain boundary strength, plane stress/strain configuration and fracture toughness on crack speed, time-to-failure, fracture behaviour and fracture morphology. Complex fracture phenomena such as crack nucleation and crack branching have been modelled without using any external fracture criterion and qualitative comparison with other numerical results has been provided. The findings of this study can be summarized as follow:

- For the configuration, specimen and conditions considered in Section 5.3.2, our PD model predicts lower values of time-to-failure and greater homogenization (lower variance) as the grain size decreases, which is in qualitative agreement with other numerical results. In contrast, the fracture behaviour (i.e. the number of occurrences) is less affected by the grain size and this is explained by the activation of a competing toughening mechanism called microcrack cloud, which is widely reported in the literature.

- The analysis described in Section 5.3.3 concluded that the GBC can affect both the time-to-failure and the peak value of crack propagation speed, which increases as the GBC decreases. In contrast, the time-to-failure increases as the GBC increases, but this is only true in a limited GBC range. Finally, GBC also affects fracture morphology with crack branching encouraged at lower values of GBC.

- In Section 5.3.4, it is observed that crack branching in polycrystalline materials is favoured at lower values of fracture toughness. Moreover, the number of branched 
cracks increases as the fracture toughness decreases, which is in qualitative agreement with other numerical results.

- The conclusions of Section 5.3.5 are such that the fracture morphology in plane strain configuration is more brittle than that in plane stress configuration, and this is in qualitative agreement with the experimental evidence.

Further improvements to the present study can be made by coupling the microstructural peridynamic model with methods such as electron backscatter diffraction [38] to measure crystal orientation with the aim to reproduce an equivalent polycrystalline model instead of producing a random texture. Secondly, experimental studies can be used to validate and refine the damage predictions of the PD model. Thirdly, an extension of the present 2D model to 3D will be considered in a subsequent paper. Fourthly, as already mentioned, adhoc studies are necessary to better understand the shielding effect captured by our PD model (Section 5.3.2) and the GBC range where the time-to-failure is effectively influenced by the value of GBC (Section 5.3.3). Lastly, structures of greater dimension could be modelled by coupling the microscopic and macroscopic PD frameworks (i.e. multiscale analysis) and by taking advantage of parallel computing, which will allow for a drastic reduction of the computational time.

\section{ACKNOWLEDGMENT}

The authors would like to acknowledge Defence Science and Technology Laboratory (Dstl) for the partial financial support. Results were obtained using the EPSRC funded ARCHIE-WeSt High Performance Computer (www.archie-west.ac.uk). EPSRC grant no. $\mathrm{EP} / \mathrm{K} 000586 / 1$. A special thanks go to the anonymous reviewers, whose time and contribution have been highly appreciated and have elevated the value of this work. 


\section{NOMENCLATURE}

\begin{tabular}{|c|c|}
\hline $\mathrm{CZM}$ & cohesive zone model \\
\hline FEM & finite element method \\
\hline $\mathrm{CCM}$ & classical continuum mechanics \\
\hline PD & peridynamics \\
\hline fcc & face-centered cubic \\
\hline bcc & body-centered cubic \\
\hline$H_{x}$ & horizon of a generic particle $\mathbf{x}$ \\
\hline$\delta$ & radius of the horizon $[\mathrm{m}]$ \\
\hline $\mathbf{f}$ & mechanical response function $\left[\mathrm{N} / \mathrm{m}^{6}\right]$ \\
\hline$c$ & bond constant $\left[\mathrm{N} / \mathrm{m}^{6}\right]$ \\
\hline$s$ & bond stretch \\
\hline$s_{0}$ & critical stretch \\
\hline $\mathbf{x}$ & vector defining the position of a generic particle $\mathbf{x}$ \\
\hline $\mathbf{x}^{\prime}$ & vector defining the position of a generic neighbour of particle $\mathbf{x}$ \\
\hline $\mathbf{y}$ & vector defining the position of particle $\mathbf{x}$ in the deformed configuration \\
\hline $\mathbf{y}^{\prime}$ & vector defining the position of particle $\mathbf{x}^{\prime}$ in the deformed configuration \\
\hline $\mathbf{b}(\mathbf{x}, t)$ & body force density field $\left[\mathrm{N} / \mathrm{m}^{3}\right]$ \\
\hline$K_{I C}$ & fracture toughness $[\mathrm{MPa} \sqrt{\mathrm{m}}]$ \\
\hline$h$ & plate's thickness [m] \\
\hline$E$ & Young's modulus $\left[\mathrm{N} / \mathrm{m}^{2}\right]$ \\
\hline$v$ & Poisson's ratio \\
\hline$G_{c}$ & critical energy release rate $[\mathrm{N} / \mathrm{m}]$ \\
\hline$C_{i j}$ & elastic moduli of the local stiffness matrix $\left[\mathrm{N} / \mathrm{m}^{2}\right]$ \\
\hline$[\mathrm{C}]$ & local stiffness matrix \\
\hline$\Delta x$ & grid spacing $[\mathrm{m}]$ \\
\hline$c_{T 1}$ & bond constant type- $1\left[\mathrm{~N} / \mathrm{m}^{6}\right]$ \\
\hline$c_{\text {T }}$ & bond constant type- $2\left[\mathrm{~N} / \mathrm{m}^{6}\right]$ \\
\hline
\end{tabular}




$\begin{array}{ll}\xi_{i j} & \text { undeformed bond length between particles } i \text { and } j[\mathrm{~m}] \\ \theta & \text { bond angle with respect to the crystal orientation angle [rad] } \\ V_{j} & \text { volume of a generic neighbouring particle } j\left[\mathrm{~m}^{3}\right] \\ q_{A} & \text { number of peridynamic bonds along A directions } \\ q_{B} & \text { number of peridynamic bonds along B directions } \\ \mathbf{u}(\mathbf{x}, t) & \text { displacement field at } \mathbf{x}[\mathrm{m}] \\ \mathbf{u}\left(\mathbf{x}^{\prime}, t\right) & \text { displacement field at } \mathbf{x}^{\prime}[\mathrm{m}] \\ \rho(\mathbf{x}) & \text { mass density at } \mathbf{x}\left[\mathrm{Kg} / \mathrm{m}^{3}\right] \\ \ddot{\mathbf{u}}(\mathbf{x}, t) & \text { acceleration vector field }\left[\mathrm{m} / \mathrm{s}^{2}\right] \\ d V_{x} & \text { volume represented by a generic neighbouring particle } \mathbf{x}^{\prime}\left[\mathrm{m}^{3}\right] \\ \mathrm{GBC} & \text { grain boundary coefficient }\end{array}$




\section{REFERENCES}

[1] I. Benedetti and M. H. Aliabadi, "A three-dimensional cohesive-frictional grainboundary micromechanical model for intergranular degradation and failure in polycrystalline materials," Comput. Methods Appl. Mech. Eng., vol. 265, pp. 36-62, 2013.

[2] M. Herbig, A. King, P. Reischig, H. Proudhon, E. M. Lauridsen, J. Marrow, J. Y. Buffire, and W. Ludwig, "3-D growth of a short fatigue crack within a polycrystalline microstructure studied using combined diffraction and phase-contrast X-ray tomography," Acta Mater., vol. 59, pp. 590-601, 2011.

[3] W. Ludwig, A. King, P. Reischig, M. Herbig, E. M. Lauridsen, S. Schmidt, H. Proudhon, S. Forest, P. Cloetens, S. R. Du Roscoat, J. Y. Buffière, T. J. Marrow, and H. F. Poulsen, "New opportunities for 3D materials science of polycrystalline materials at the micrometre lengthscale by combined use of X-ray diffraction and Xray imaging," Mater. Sci. Eng. A, vol. 524, pp. 69-76, 2009.

[4] M. A. Groeber, B. K. Haley, M. D. Uchic, D. M. Dimiduk, and S. Ghosh, "3D reconstruction and characterization of polycrystalline microstructures using a FIBSEM system," Mater. Charact., vol. 57, pp. 259-273, 2006.

[5] M. Paggi and P. Wriggers, "Stiffness and strength of hierarchical polycrystalline materials with imperfect interfaces," J. Mech. Phys. Solids, vol. 60, no. 4, pp. 557-572, 2012.

[6] H. D. Espinosa and P. D. Zavattieri, "A grain level model for the study of failure initiation and evolution in polycrystalline brittle materials. Part I: Theory and numerical implementation," vol. 35, pp. 333-364, 2003.

[7] H. D. Espinosa and P. D. Zavattieri, "A grain level model for the study of failure initiation and evolution in polycrystalline brittle materials. Part II: Numerical examples," Mech. Mater., vol. 35, pp. 365-394, 2003.

[8] J. Zhai, V. Tomar, and M. Zhou, "Micromechanical Simulation of Dynamic Fracture Using the Cohesive Finite Element Method," J. Eng. Mater. Technol., vol. 126, p. 179, 2004.

[9] N. Sukumar, D. J. Srolovitz, T. J. Baker, and J. H. Prévost, "Brittle fracture in polycrystalline microstructures with the extended finite element method," Int. $J$. Numer. Methods Eng., vol. 56, pp. 2015-2037, 2003.

[10] G. K. Sfantos and M. H. Aliabadi, "Multi-scale boundary element modelling of material degradation and fracture," Comput. Methods Appl. Mech. Eng., vol. 196, pp. 1310-1329, 2007.

[11] G. K. Sfantos and M. H. Aliabadi, "A boundary cohesive grain element formulation for modelling intergranular microfracture in polycrystalline brittle materials," Int. J. Numer. Methods Eng., vol. 69, pp. 1590-1626, 2007. 
[12] I. Benedetti and M. H. Aliabadi, "A grain boundary formulation for the analysis of three-dimensional polycrystalline microstructures," Key Eng. Mater., vol. 525-526, pp. 1-4, 2012.

[13] I. Benedetti and M. H. Aliabadi, "A three-dimensional grain boundary formulation for microstructural modeling of polycrystalline materials," Comput. Mater. Sci., vol. 67, pp. 249-260, 2013.

[14] A. G. Crocker, P. E. J. Flewitt, and G. E. Smith, "Computational modelling of fracture in polycrystalline materials," Int. Mater. Rev., vol. 50, no. 2, pp. 99-125, 2005.

[15] E. Madenci and E. Oterkus, Peridynamic theory and its applications. 2014.

[16] G. Zi, T. Rabczuk, and W. Wall, "Extended meshfree methods without branch enrichment for cohesive cracks," Comput. Mech., vol. 40, pp. 367-382, 2007.

[17] T. L. Anderson, Fracture Mechanics - Fundamentals and Applications, 3rd ed. Boca Raton: Taylor \& Francis, 2005.

[18] S. A. Silling, "Reformulation of elasticity theory for discontinuities and long-range forces," J. Mech. Phys. Solids, vol. 48, pp. 175-209, 2000.

[19] E. Askari, F. Bobaru, R. B. Lehoucq, M. L. Parks, S. A. Silling, and O. Weckner, "Peridynamics for multiscale materials modeling," J. Phys. Conf. Ser., vol. 125, p. 012078, 2008.

[20] M. Ghajari, L. Iannucci, and P. Curtis, "A peridynamic material model for the analysis of dynamic crack propagation in orthotropic media," Comput. Methods Appl. Mech. Eng., vol. 276, pp. 431-452, 2014.

[21] P. Seleson and M. Parks, "On the role of the influence function in the peridynamic theory," Int. J. Multiscale Comput. Eng., vol. 9, no. 6, pp. 689-706, 2011.

[22] E. Oterkus and E. Madenci, "Peridynamic analysis of fiber-reinforced composite materials," J. Mech. Mater. Struct., vol. 7, no. 1, 2012.

[23] Y. Hirose and T. Mura, "Nucleation mechanism of stress corrosion cracking from notches,” Eng. Fract. Mech., vol. 19, no. 2, pp. 317-329, 1984.

[24] J. J. Rimoli and M. Ortiz, "A three-dimensional multiscale model of intergranular hydrogen-assisted cracking," Philos. Mag., vol. 90, no. 917270850, pp. 2939-2963, 2010 .

[25] W. F. Hosford, The mechanics of crystals and textured polycrystals. New York, USA: Oxford University Press, 1993.

[26] J. J. Rimoli, "A computational model for intergranular stress corrosion cracking," 2009.

[27] B. Lawn, Fracture of Brittle Solids, 2nd ed. Cambridge University Press, 1993. 
[28] D. K. M. Shum and J. W. Hutchinson, "On toughening by microcracks," Mechanics of Materials, vol. 9. pp. 83-91, 1990.

[29] J. W. Hutchinson, "Mechanisms of toughening in ceramics," Theoretical and applied mechanics. pp. 139-144, 1989.

[30] M. Ruhle, A. G. Evans, R. M. McMeeking, P. G. Charalambides and J. W. Hutchinson, "Microcrack toughening in alumina/zirconia," Acta Met., vol. 35, no. 11, pp. 2701-2710, 1987.

[31] Y. Toi and S. N. Atluri, "Finite element analysis of static and dynamic fracture of brittle microcracking solids. Part 1: Formulation and simple numerical examples," Int. J. Plast., vol. 6, pp. 166-188, 1990.

[32] E. Johnson, "Simulations of microcracking in the process region of ceramics with a cell model," Int. J. Fract., vol. 111, no. 1978, pp. 361-380, 2001.

[33] H. Wang, Z. Liu, D. Xu, Q. Zeng, and Z. Zhuang, "Extended finite element method analysis for shielding and amplification effect of a main crack interacted with a group of nearby parallel microcracks," Int. J. Damage Mech., vol. 25, no. 1, pp. 4-25, 2016.

[34] K. R. Chandar and W. G. Knauss, "Dynamic crack-tip stresses under stress wave loading -A comparison of theory and experiment," Int. J. Fract., vol. 20, pp. 209-222, 1982.

[35] F. Bobaru and W. Hu, "The meaning, selection, and use of the peridynamic horizon and its relation to crack branching in brittle materials," Int. J. Fract., vol. 176, pp. 215-222, 2012.

[36] Y. D. Ha and F. Bobaru, "Studies of dynamic crack propagation and crack branching with peridynamics," Int. J. Fract., vol. 162, pp. 229-244, 2010.

[37] Y. D. Ha and F. Bobaru, "Characteristics of dynamic brittle fracture captured with peridynamics," Eng. Fract. Mech., vol. 78, no. 6, pp. 1156-1168, 2011.

[38] E. Pouillier, A. F. Gourgues, D. Tanguy, and E. P. Busso, "A study of intergranular fracture in an aluminium alloy due to hydrogen embrittlement," Int. J. Plast., vol. 34, pp. 139-153, 2012.

[39] S. A. Silling and E. Askari, "A meshfree method based on the peridynamic model of solid mechanics," Comput. Struct., vol. 83, pp. 1526-1535, 2005. 


\section{APPENDIX A}

The aim of this appendix is to describe the derivation of the expression for type- 1 bond constant $c_{T 1}$ and type- 2 bond constant $c_{T 2}$ used in the peridynamic micro-mechanical model for cubic crystals.

As already shown in Section 4 , three independent material constants, namely $\mathrm{C}_{11}, \mathrm{C}_{12}$ and $\mathrm{C}_{44}$, are necessary to fully describe the micro-mechanical response of the cubic crystal. Therefore, three independent peridynamic material constants would be necessary. Nevertheless, for the sake of simplicity, the current peridynamic micro-mechanical model is described by two independent material constants only, namely $c_{T 1}$ and $c_{T 2}$. According to the procedure, a body under study is subjected to three independent loading conditions. The resulting PD and CCM strain energy density are equated and the solution of the resulting equations leads to the PD micro-mechanical properties $c_{T 1}$ and $c_{T 2}$. The following three subsections describe in detail the three different loading conditions and the relevant calculations.

\section{First loading condition}

The first loading condition consists in the application of a constant strain along direction-1 which, in this particular case, is equal to direction- $x$. (Fig. A.1):

$$
\varepsilon_{11}=\zeta=0.001 \quad \varepsilon_{22}=0.0 \quad \gamma_{12}=0.0
$$

Since $\zeta<<1, \quad \cos (\Delta \theta) \cong \cos (0)=1$ and $\sin (\Delta \theta) \cong \sin (0)=0$. By means of simple geometrical considerations, the bond length in the deformed configuration and the stretch can be calculated as shown in Fig. A.1:

$$
\begin{array}{r}
l_{\text {def }}=l_{\text {undef }}+\zeta \cdot \xi_{x} \cdot \cos (\theta)=l_{\text {undef }} \cdot\left(1+\zeta \cdot \cos ^{2}(\theta)\right) \\
s=\frac{\Delta l}{l_{\text {undef }}}=\frac{l_{\text {def }}-l_{\text {undef }}}{l_{\text {undef }}}=\zeta \cdot \cos ^{2}(\theta)
\end{array}
$$

As described in [39], the PD strain energy density associated with a generic material point can be written as: 


$$
W_{P D}=\frac{1}{2} \cdot \int_{H_{x}} w \cdot d V
$$

The micro-potential function $w$ represents the strain energy accumulated in a single peridynamic bond due to the application of external loads on the body. It can be calculated by using the analogy of the well-known formula for the strain energy stored in a tensioned single spring as:

$$
w=\frac{1}{2} \cdot c \cdot s^{2} \cdot l_{\text {undef }}
$$

Considering a neighbourhood $H_{x}$ of disk shape, whose thickness equals the thickness of the plate $h$, the infinitesimal volume has the following expression:

$$
d V=(\xi \cdot d \theta) \cdot d \xi \cdot h
$$

Considering the contribution of both type- 1 and type- 2 bond constants, the expression of the peridynamic strain energy density for a generic material point $i$ can be written as follows:

$$
\begin{aligned}
W_{P D}(i)= & \frac{1}{4} \cdot c_{T 1} \cdot h \cdot \zeta^{2} \cdot \int_{0}^{\delta} \xi^{2} \cdot d \xi \cdot \int_{0}^{2 \cdot \pi} \cos ^{4}(\theta) d \theta+ \\
& \frac{1}{4} \cdot c_{T 2} \cdot \zeta^{2} \cdot \cos ^{4}\left(\vartheta_{A}\right) \cdot \sum_{j=1}^{q A} \xi_{i j} \cdot V_{j}+ \\
& \frac{1}{4} \cdot c_{T 2} \cdot \zeta^{2} \cdot \cos ^{4}\left(\vartheta_{B}\right) \cdot \sum_{j=1}^{q B} \xi_{i j} \cdot V_{j}
\end{aligned}
$$

Consider the definition of the following parameters $\beta_{A}$ and $\beta_{B}$ :

$$
\begin{aligned}
& \beta_{A}=\sum_{j=1}^{q_{A}} \xi_{i j} V_{j} \\
& \beta_{B}=\sum_{j=1}^{q_{B}} \xi_{i j} V_{j}
\end{aligned}
$$


where subscript $A$ is associated with directions $\theta=\frac{\pi}{4}, \frac{5}{4} \pi$, whilst subscript $B$ is associated with directions $\theta=\frac{3}{4} \pi, \frac{7}{4} \pi$. In Eq. (A.7) and in Eq. (A.8), $i$ and $j$ refer to a generic particle and its neighbour respectively, $V_{j}$ denotes the volume of particle $j, \xi_{i j}$ is the initial length of the bond between particles $i$ and $j$, and $q_{A}$ and $q_{B}$ represent the number of PD bonds along the directions associated with $A$ and $B$, respectively.

By exploiting the definition of $\beta_{A}$ and $\beta_{B}$, the PD strain energy density can be rewritten as:

$$
\begin{aligned}
W_{P D}(i)= & \frac{1}{4} \cdot c_{T 1} \cdot h \cdot \zeta^{2} \cdot \frac{\delta^{3}}{3} \cdot \frac{3}{4} \cdot \pi+ \\
& \frac{1}{4} \cdot c_{T 2} \cdot \zeta^{2} \cdot 0.25 \cdot \beta_{A}+ \\
& \frac{1}{4} \cdot c_{T 2} \cdot \zeta^{2} \cdot 0.25 \cdot \beta_{B}
\end{aligned}
$$

With few more simplifications, the final expression can be obtained as:

$$
W_{P D}(i)=\frac{1}{4} \cdot c_{T 1} \cdot h \cdot \zeta^{2} \cdot \frac{\delta^{3}}{3} \cdot \frac{3}{4} \cdot \pi+\frac{1}{4} \cdot c_{T 2} \cdot \zeta^{2} \cdot 0.25 \cdot\left(\beta_{A}+\beta_{B}\right)
$$

The next step is to write the expression of the strain energy density according to CCM. In the case of a model with a two-dimensional simplification, the reduced global stiffness matrix $[\mathrm{Q}]$ of a cubic crystal can be written as:

$$
\left[\begin{array}{c}
\sigma_{11} \\
\sigma_{22} \\
\tau_{12}
\end{array}\right]=\left[\begin{array}{ccc}
Q_{11} & Q_{12} & 0 \\
Q_{12} & Q_{11} & 0 \\
0 & 0 & Q_{44}
\end{array}\right] \cdot\left[\begin{array}{c}
\varepsilon_{11} \\
\varepsilon_{22} \\
\gamma_{12}
\end{array}\right]
$$

According to CCM, the expression of strain energy density can be written as:

$$
W_{C C M}=\frac{1}{2} \cdot\left(\sigma_{11} \cdot \varepsilon_{11}+\sigma_{22} \cdot \varepsilon_{22}+\tau_{12} \cdot \gamma_{12}\right)
$$

This can be rewritten for the first loading condition as: 


$$
W_{C C M}=\frac{1}{2} \cdot \sigma_{11} \cdot \varepsilon_{11}=\frac{1}{2} \cdot Q_{11} \cdot \varepsilon_{11}^{2}=\frac{1}{2} \cdot Q_{11} \cdot \zeta^{2}
$$

The first equation of our system is obtained by equating PD and CCM strain energy density expressions:

$$
\frac{1}{16} \cdot c_{T 1} \cdot h \cdot \zeta^{2} \cdot \delta^{3} \cdot \pi+\frac{1}{4} \cdot c_{T 2} \cdot \zeta^{2} \cdot 0.25 \cdot\left(\beta_{A}+\beta_{B}\right)=\frac{1}{2} \cdot Q_{11} \cdot \zeta^{2}
$$

\section{Second loading condition}

The second loading condition consists in the application of a constant strain along both direction-1 and direction-2 (Fig. A.2). In this particular case, direction-1 is equal to direction$x$ and direction-2 is equal to direction- $y$.

$$
\varepsilon_{11}=\zeta=0.001 \quad \varepsilon_{22}=\zeta=0.001 \quad \gamma_{12}=0.0
$$

By means of simple geometrical considerations, it is possible to derive the following expression for the generic bond length and stretch in the deformed configuration:

$$
\begin{aligned}
l_{\text {def }} & =l_{\text {undef }}+l_{\text {undef }} \cdot \zeta \cdot\left(\sin ^{2}(\theta)+\cos ^{2}(\theta)\right) \\
s & =\frac{l_{\text {def }}-l_{\text {undef }}}{l_{\text {undef }}}=\zeta \cdot\left(\sin ^{2}(\theta)+\cos ^{2}(\theta)\right)
\end{aligned}
$$

Similar to (A.6), the PD strain energy density can be written as:

$$
\begin{aligned}
W_{P D}(i)= & \frac{1}{4} \cdot c_{T 1} \cdot h \cdot \zeta^{2} \cdot \int_{0}^{\delta} \xi^{2} \cdot d \xi \cdot\left(\int_{0}^{2 \cdot \pi} \cos ^{4}(\theta) d \theta+\int_{0}^{2 \cdot \pi} \sin ^{4}(\theta) d \theta+2 \cdot \int_{0}^{2 \cdot \pi} \cos ^{2}(\theta) \cdot \sin ^{2}(\theta) d \theta\right)+ \\
& +\frac{1}{4} \cdot c_{T 2} \cdot \zeta^{2} \cdot\left(\cos ^{4}\left(\vartheta_{A}\right)+\sin ^{4}\left(\vartheta_{A}\right)+2 \cdot \cos ^{2}\left(\vartheta_{A}\right) \cdot \cos ^{2}\left(\vartheta_{A}\right)\right) \cdot \beta_{A}+ \\
& +\frac{1}{4} \cdot c_{T 2} \cdot \zeta^{2} \cdot\left(\cos ^{4}\left(\vartheta_{B}\right)+\sin ^{4}\left(\vartheta_{B}\right)+2 \cdot \cos ^{2}\left(\vartheta_{B}\right) \cdot \cos ^{2}\left(\vartheta_{B}\right)\right) \cdot \beta_{B}
\end{aligned}
$$

This can be further simplified as:

$$
W_{P D}(i)=\frac{1}{4} \cdot c_{T 1} \cdot h \cdot \zeta^{2} \cdot \frac{\delta^{3}}{3} \cdot 2 \pi+\frac{1}{4} \cdot c_{T 2} \cdot \zeta^{2} \cdot\left(\beta_{A}+\beta_{B}\right)
$$


Recalling (A.12), CCM strain energy density can be written for this loading condition as:

$$
W_{C C M}=\frac{1}{2} \cdot\left(\sigma_{11} \cdot \varepsilon_{11}+\sigma_{22} \cdot \varepsilon_{22}\right)=\sigma_{11} \cdot \varepsilon_{11}=\left(Q_{11}+Q_{12}\right) \cdot \zeta^{2}
$$

By equating PD and CCM expressions, the second equation of our system can be written as:

$$
\frac{1}{6} \cdot c_{T 1} \cdot h \cdot \zeta^{2} \cdot \delta^{3} \cdot \pi+\frac{1}{4} \cdot c_{T 2} \cdot \zeta^{2} \cdot\left(\beta_{A}+\beta_{B}\right)=\left(Q_{11}+Q_{12}\right) \cdot \zeta^{2}
$$

\section{Third loading condition}

The third loading condition consists of the application of a constant simple shear strain (Fig. A.3):

$$
\varepsilon_{11}=0.0 \quad \varepsilon_{22}=0.0 \quad \gamma_{12}=0.001=\zeta
$$

Since $\zeta<<1, \quad \cos (\Delta \theta) \cong \cos (0)=1$ and $\sin (\Delta \theta) \cong \sin (0)=0$. By means of simple geometrical considerations, the bond length and stretch in the deformed configuration can be calculated as:

$$
\begin{gathered}
l_{\text {def }}=l_{\text {undef }} \cdot(1+\zeta \cdot \cos (\theta) \cdot \sin (\theta)) \\
s=\frac{\Delta l}{l_{\text {undef }}}=\frac{l_{\text {def }}-l_{\text {undef }}}{l_{\text {undef }}}=\zeta \cdot \cos (\theta) \cdot \sin (\theta)
\end{gathered}
$$

The PD strain energy density for this loading condition can be written as:

$$
\begin{aligned}
W_{P D}(i)= & \frac{1}{4} \cdot c_{T 1} \cdot h \cdot \zeta^{2} \cdot \int_{0}^{\delta} \xi^{2} \cdot d \xi \cdot \int_{0}^{2 \cdot \pi} \cos ^{2}(\theta) \cdot \sin ^{2}(\theta) \cdot d \theta+ \\
& +\frac{1}{4} \cdot c_{T 2} \cdot \zeta^{2} \cdot \cos ^{2}\left(\vartheta_{A}\right) \cdot \sin ^{2}\left(\vartheta_{A}\right) \cdot \beta_{A}+ \\
& \left.+\frac{1}{4} \cdot c_{T 2} \cdot \zeta^{2} \cdot \cos ^{2}\left(\vartheta_{B}\right) \cdot \sin ^{2}\left(\vartheta_{B}\right)\right) \cdot \beta_{B}
\end{aligned}
$$

The calculation of the integrals leads to the following expression:

$$
W_{P D}(i)=\frac{1}{4} \cdot c_{T 1} \cdot h \cdot \zeta^{2} \cdot \frac{\delta^{3}}{3} \cdot \frac{\pi}{4}+\frac{1}{4} \cdot c_{T 2} \cdot \zeta^{2} \cdot \frac{1}{4}\left(\beta_{A}+\beta_{B}\right)
$$


With few more simplification, it can be rewritten as:

$$
W_{P D}(i)=\frac{1}{48} \cdot c_{T 1} \cdot h \cdot \zeta^{2} \cdot \delta^{3} \cdot \pi+\frac{1}{16} \cdot c_{T 2} \cdot \zeta^{2} \cdot\left(\beta_{A}+\beta_{B}\right)
$$

Recalling (A.12), CCM strain energy density for this loading condition can be written as:

$$
W_{C C M}=\frac{1}{2} \cdot\left(\tau_{12} \cdot \gamma_{12}\right)=\frac{1}{2} \cdot Q_{44} \cdot \zeta^{2}
$$

By equating PD and CCM strain energy density, the third equation of the system can be written as:

$$
\frac{1}{2} \cdot Q_{44} \cdot \zeta^{2}=\frac{1}{48} \cdot c_{T 1} \cdot h \cdot \zeta^{2} \cdot \delta^{3} \cdot \pi+\frac{1}{16} \cdot c_{T 2} \cdot \zeta^{2} \cdot\left(\beta_{A}+\beta_{B}\right)
$$

The solution of the system constituted by the three equations (A.14), (A.20) and (A.27) leads to the following expressions for the peridynamic micro-mechanical material parameters:

$$
c_{T 1}=\frac{12 \cdot\left(Q_{11}-Q_{44}\right)}{\pi \cdot h \cdot \delta^{3}} \quad, \quad c_{T 2}=\frac{4 \cdot\left(3 \cdot Q_{44}-Q_{11}\right)}{\beta_{A}+\beta_{B}} \quad, \quad Q_{44}=Q_{12}
$$

In case of plane stress configuration:

$$
Q_{11}=\frac{c_{11}^{2}-c_{12}^{2}}{c_{11}} \quad, \quad Q_{12}=\frac{c_{11} c_{12}-c_{12}^{2}}{c_{11}}
$$

Resulting in:

$$
c_{T 1}=\frac{12\left(c_{11}^{2}-c_{11} c_{12}\right)}{\pi h \delta^{3} c_{11}} \quad, \quad c_{T 2}=\frac{4\left(3 c_{11} c_{12}-2 c_{12}^{2}-c_{11}^{2}\right)}{\left(\beta_{A}+\beta_{B}\right) c_{11}}
$$

In case of plane strain configuration:

$$
Q_{11}=c_{11} \quad, \quad Q_{12}=c_{12}
$$

Resulting in: 


$$
c_{T 1}=\frac{12 \cdot\left(c_{11}-c_{12}\right)}{\pi \cdot h \cdot \delta^{3}} \quad, \quad c_{T 2}=\frac{4 \cdot\left(3 \cdot c_{12}-c_{11}\right)}{\beta_{A}+\beta_{B}}
$$

Since the quantities $\beta_{A}$ and $\beta_{B}$ depend on the grain orientation $\gamma$, the mechanical behaviour of each crystal is potentially different with respect to that of its neighbouring crystals. 


\section{Figures}

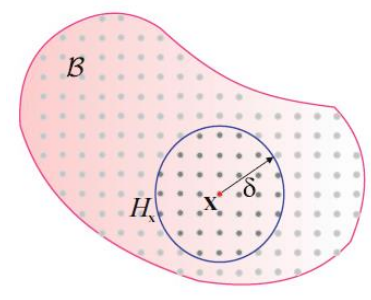

Fig. 1. Peridynamic interactions.



Fig. 2. Definition of bond constant and critical stretch for linear elastic brittle material.

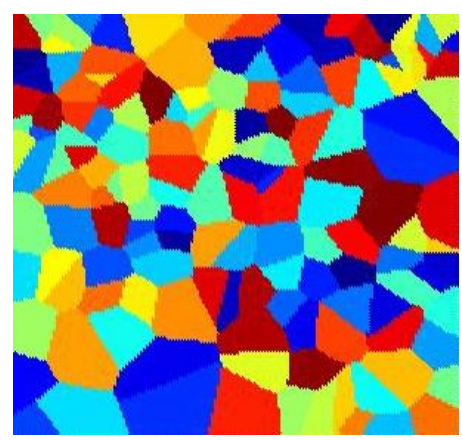

Fig. 3. Polycrystalline material model. 


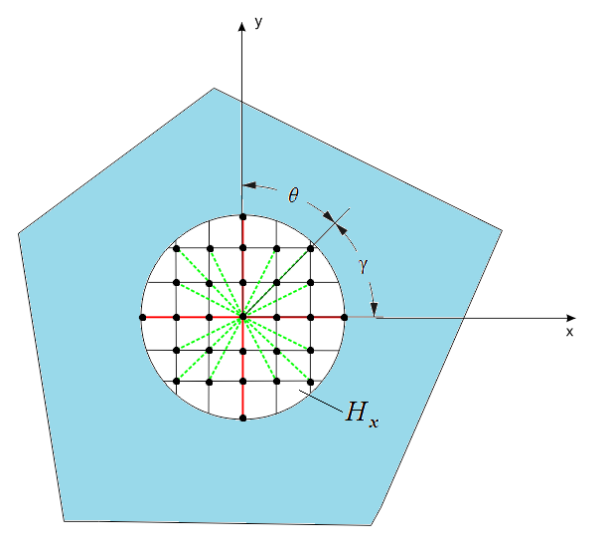

Fig. 4. Type-1 (dashed green lines) and type-2 (solid red lines) bonds for the peridynamic micromechanical model.

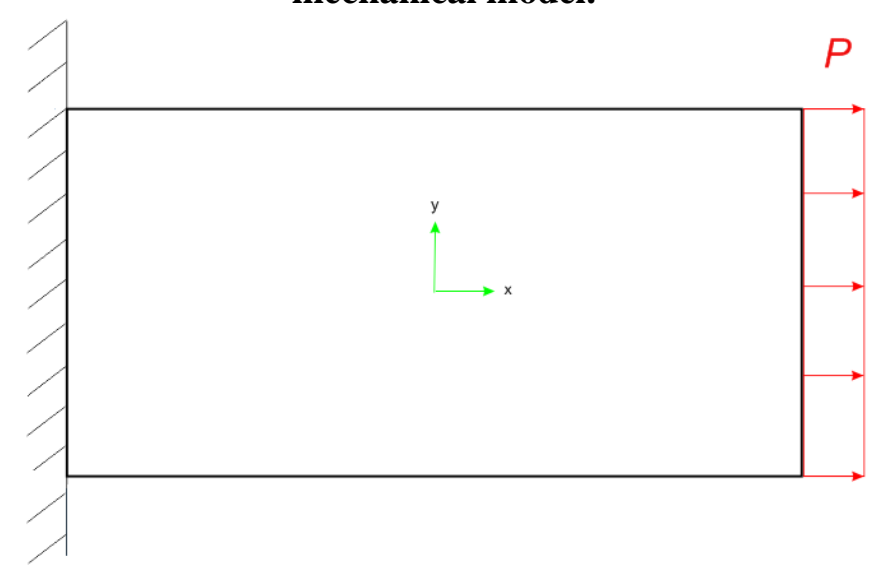

Fig. 5. Iron crystal for static analysis.
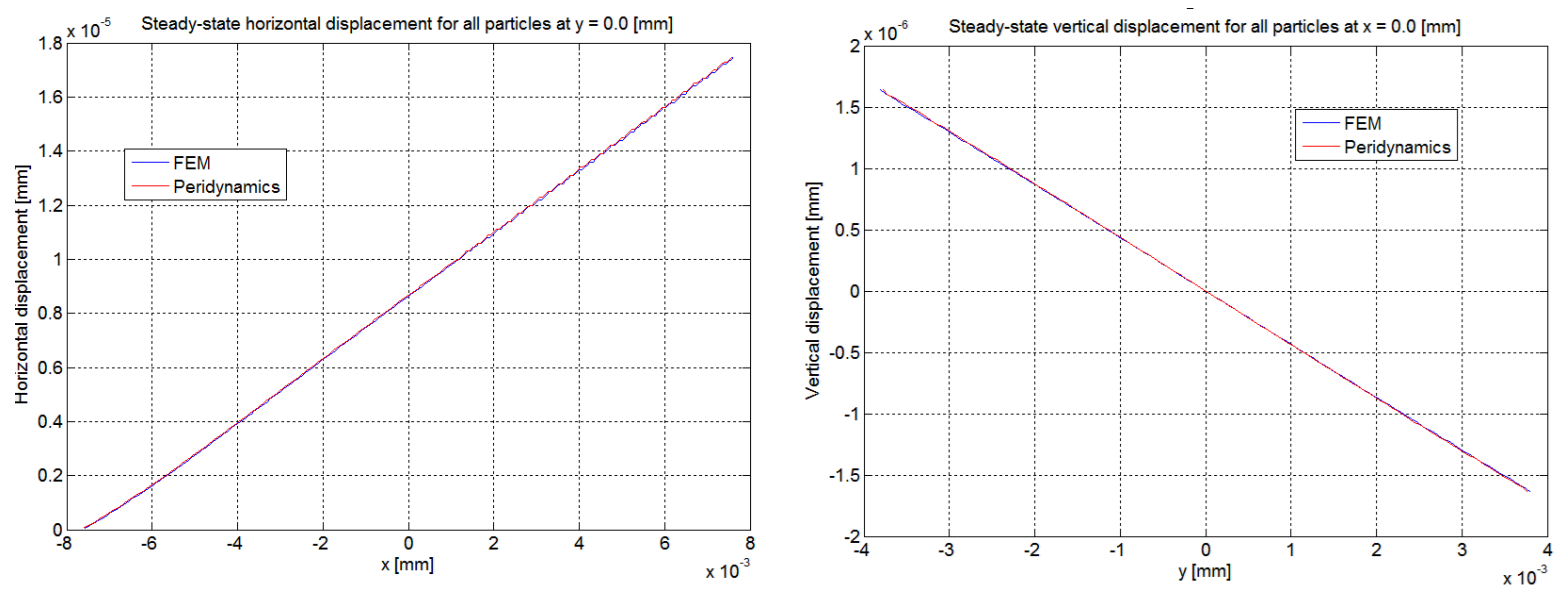

Fig. 6. Displacement field comparison between FEM and PD for the iron crystal in plane stress configuration and $0^{\circ}$ orientation. 

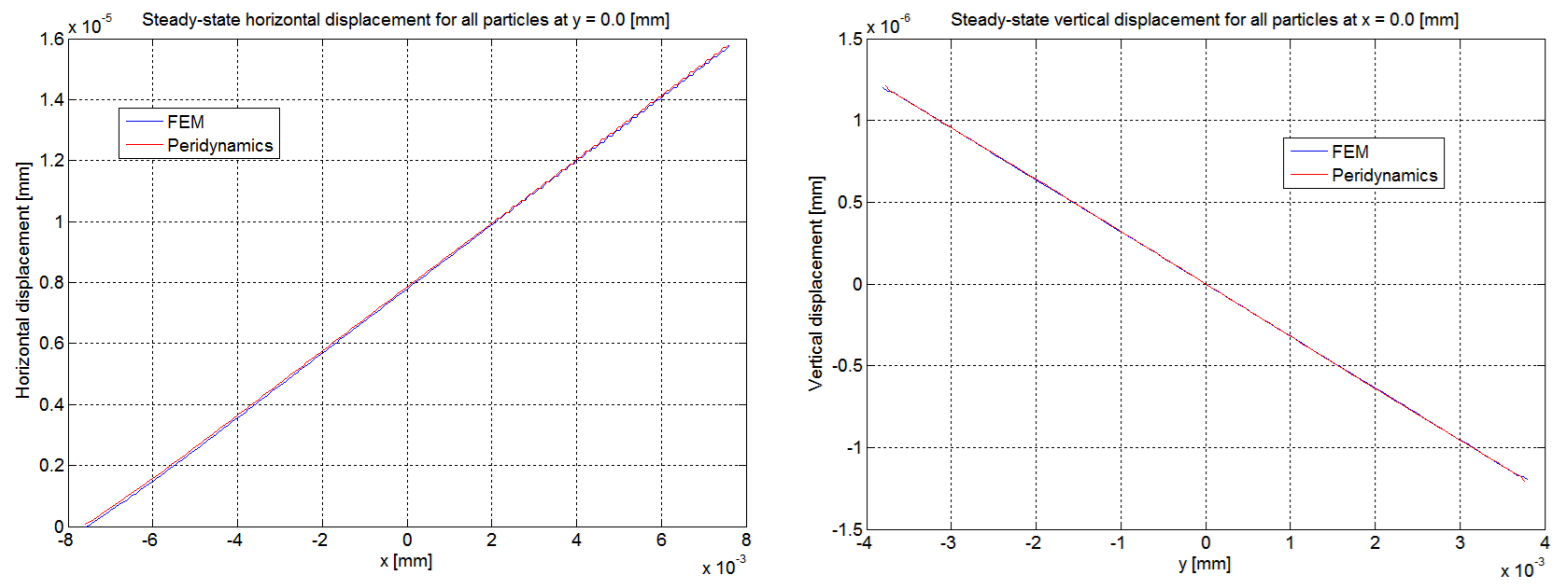

Fig. 7. Displacement field comparison between FEM and PD for the iron crystal in plane stress configuration and $45^{\circ}$ orientation.


Fig. 8. Displacement field comparison between FEM and PD for the iron crystal in plane strain configuration and $0^{\circ}$ orientation.
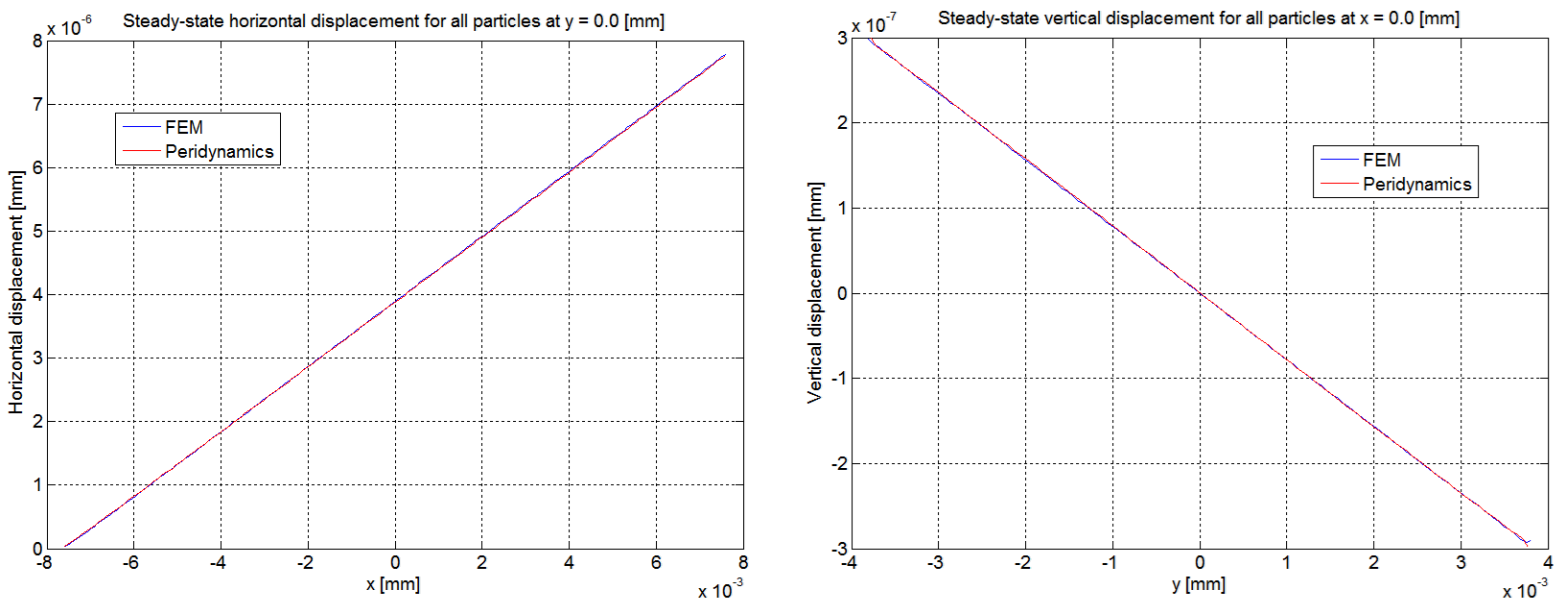

Fig. 9. Displacement field comparison between FEM and PD for the iron crystal in plane strain configuration and $45^{\circ}$ orientation. 


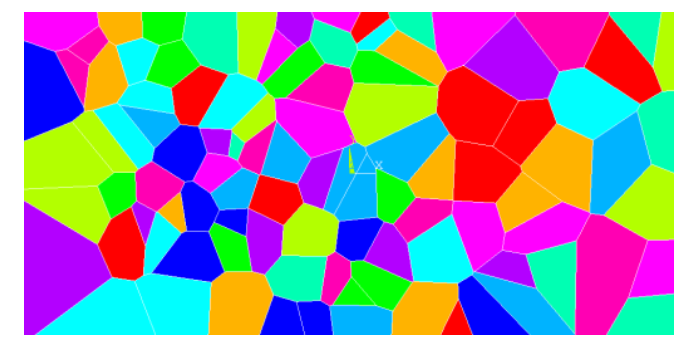

Fig. 10. Iron polycrystal considered for static analysis.
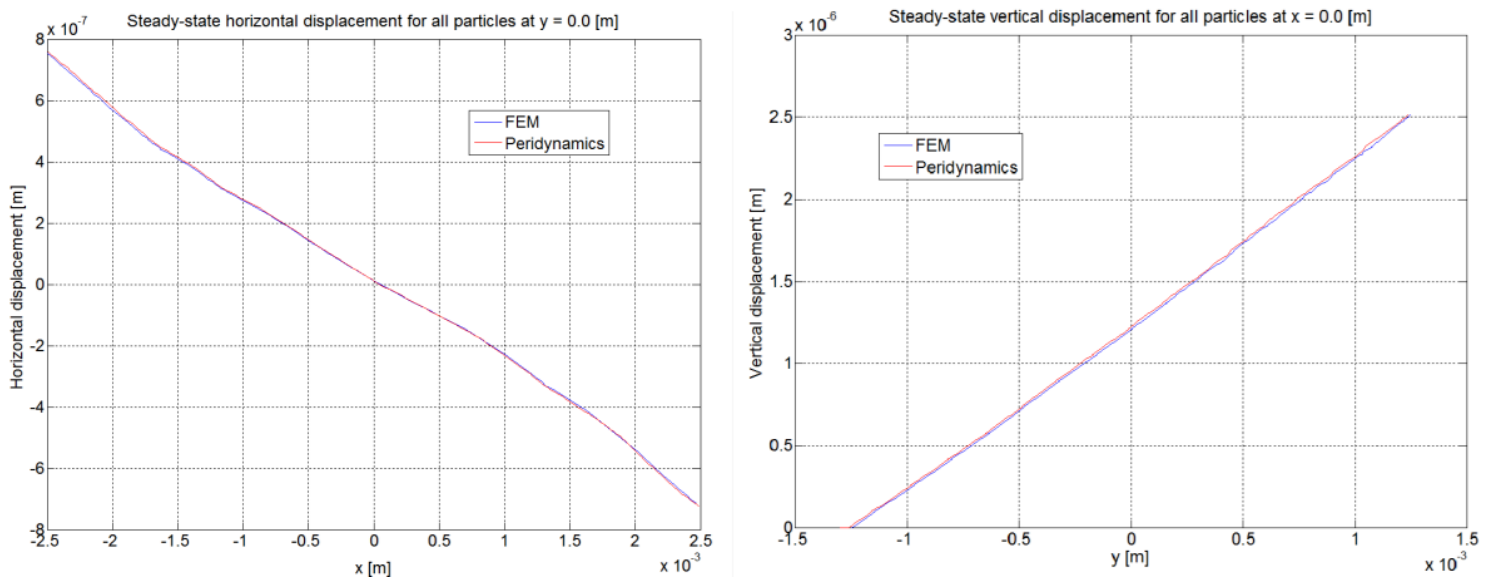

Fig. 11. Displacement field comparison between FEM and PD for the iron polycrystal in plane stress configuration.
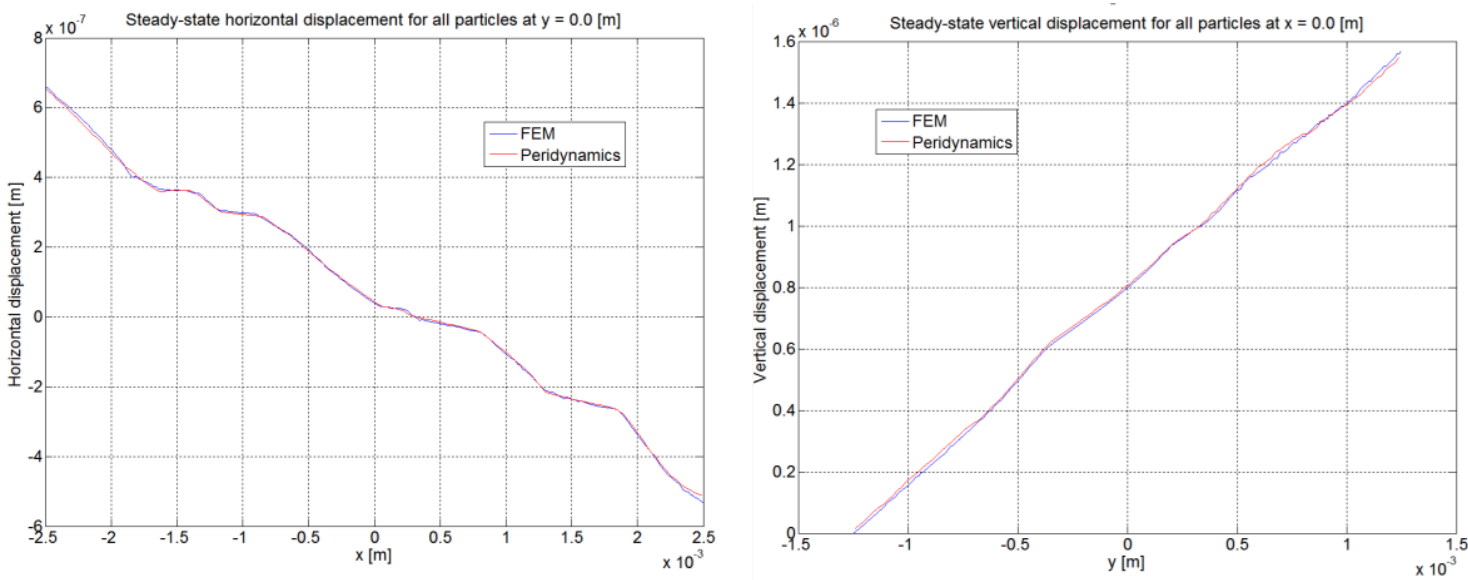

Fig. 12. Displacement field comparison between FEM and PD for the iron polycrystal in plane strain configuration.

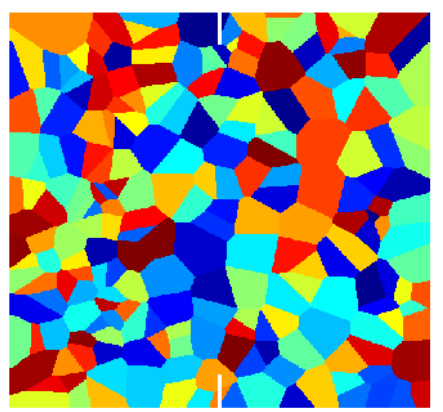

Fig. 13. AISI 4340 polycrystal for convergence analysis ( 225 grains). 


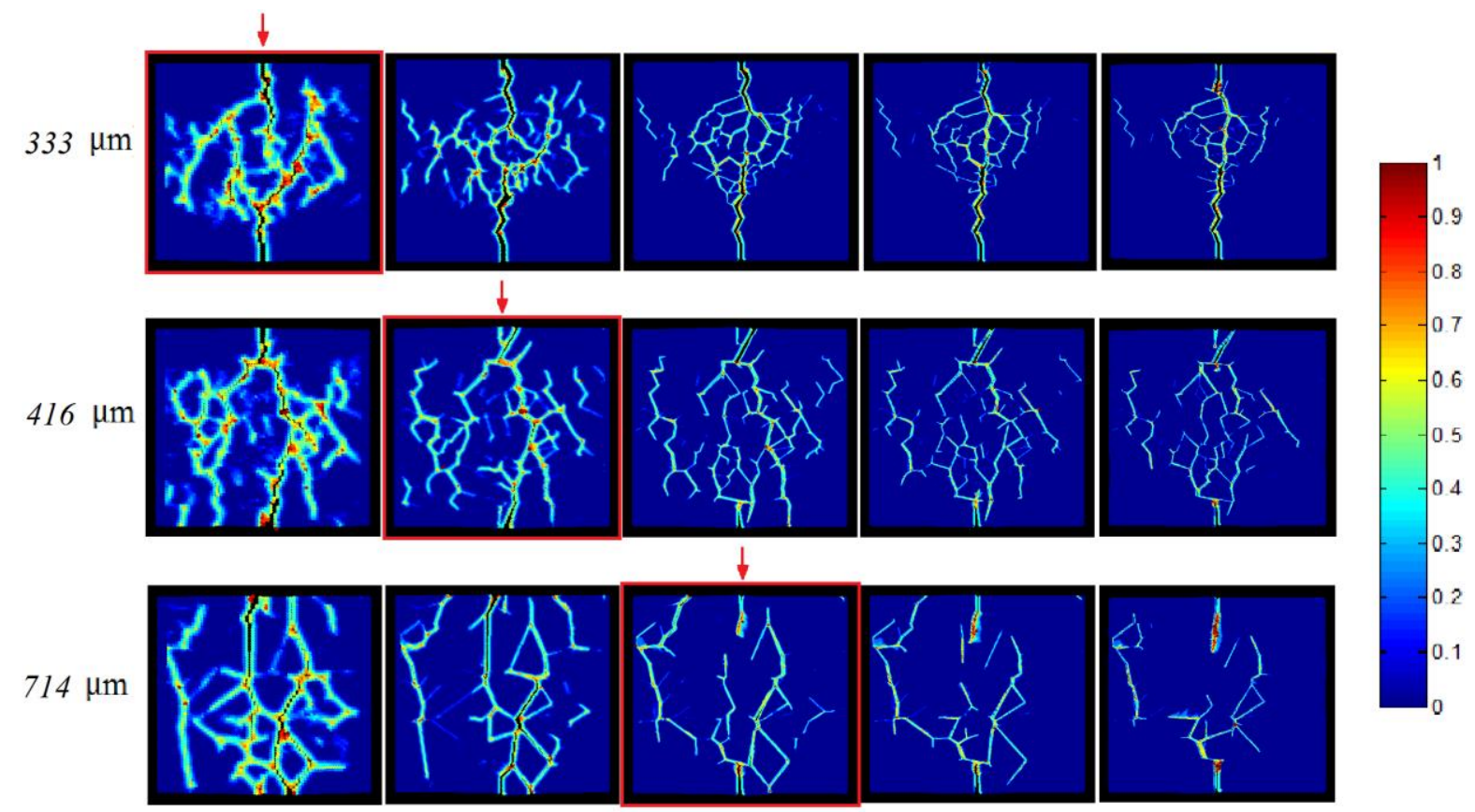

Fig. 14. GBC $=0.5$, time $=2.4 \mu$ s. Fracture pattern comparison of three polycrystals with different average grain size $(333 \mu \mathrm{m}, 416 \mu \mathrm{m}$ and $714 \mu \mathrm{m})$ and five different horizon values: from left to right, $202.7 \mu \mathrm{m}$ ( $74 \times 74$ particles), $100 \mu \mathrm{m}$ (150 x 150 particles), $50 \mu \mathrm{m}(300 \times 300$ particles $), 37.5 \mu \mathrm{m}(400 \times 400$ particles $)$ and $30 \mu \mathrm{m}(500 \times 500$ particles $)$.

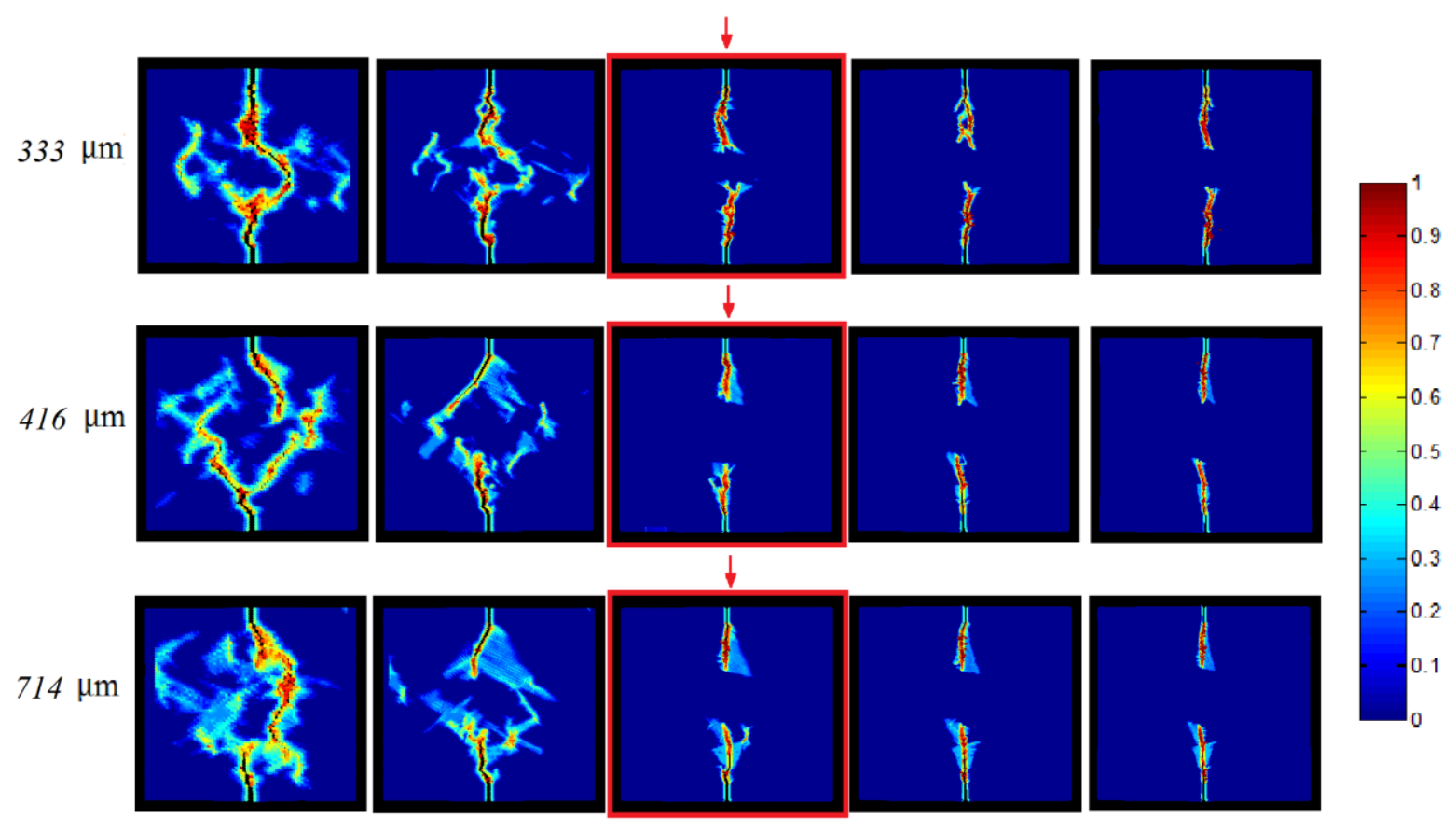

Fig. 15. GBC $=1$, time $=2.4 \mu \mathrm{s}$. Fracture pattern comparison of three polycrystals with different average grain size $(333 \mu \mathrm{m}, 416 \mu \mathrm{m}$ and $714 \mu \mathrm{m})$ and five different horizon values: from left to right, $202.7 \mu \mathrm{m}$ (74 x 74 particles), $100 \mu \mathrm{m}$ (150 x 150 particles), $50 \mu \mathrm{m}$ (300 x 300 particles), 37.5 $\mu \mathrm{m}(400 \times 400$ particles $)$ and $30 \mu \mathrm{m}(500 \times 500$ particles $)$. 




Fig. 16. $\mathrm{GBC}=2$, time $=2.4 \mu \mathrm{s}$. Fracture pattern comparison of three polycrystals with different average grain size $(333 \mu \mathrm{m}, 416 \mu \mathrm{m}$ and $714 \mu \mathrm{m})$ and five different horizon values: from left to right, $202.7 \mu \mathrm{m}$ (74 x 74 particles), $100 \mu \mathrm{m}$ (150 x 150 particles), $50 \mu \mathrm{m}$ (300 x 300 particles), 37.5 $\mu \mathrm{m}(400 \times 400$ particles $)$ and $30 \mu \mathrm{m}(500 \times 500$ particles $)$.

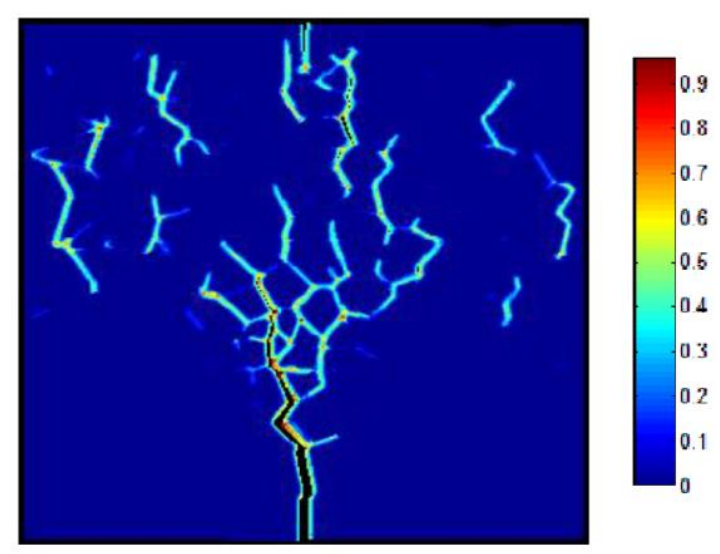

Fig. 17. Meaning of occurrence: occurrence (bottom notch), non-occurrence (top notch). 


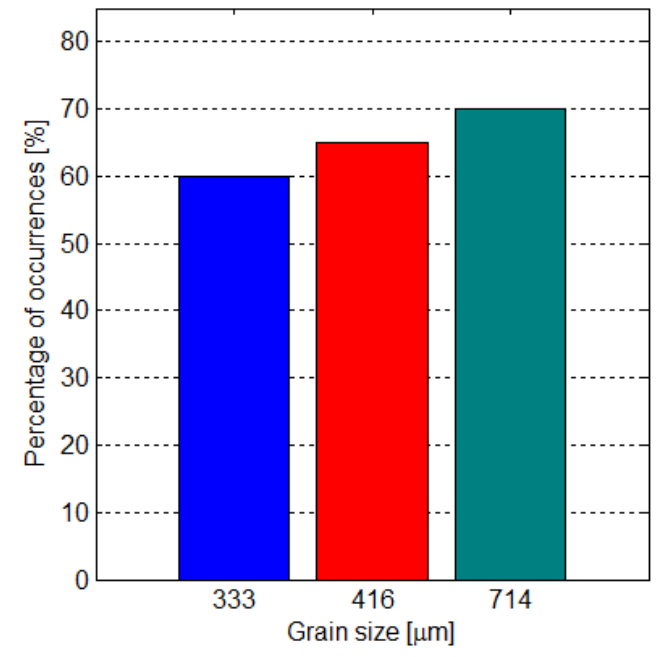

Fig. 18. Grain size effect on the number of occurrences.

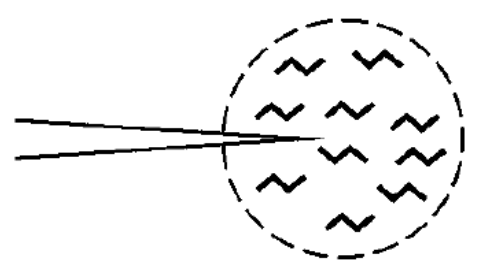

Fig. 19. Microcrack cloud mechanism [27].

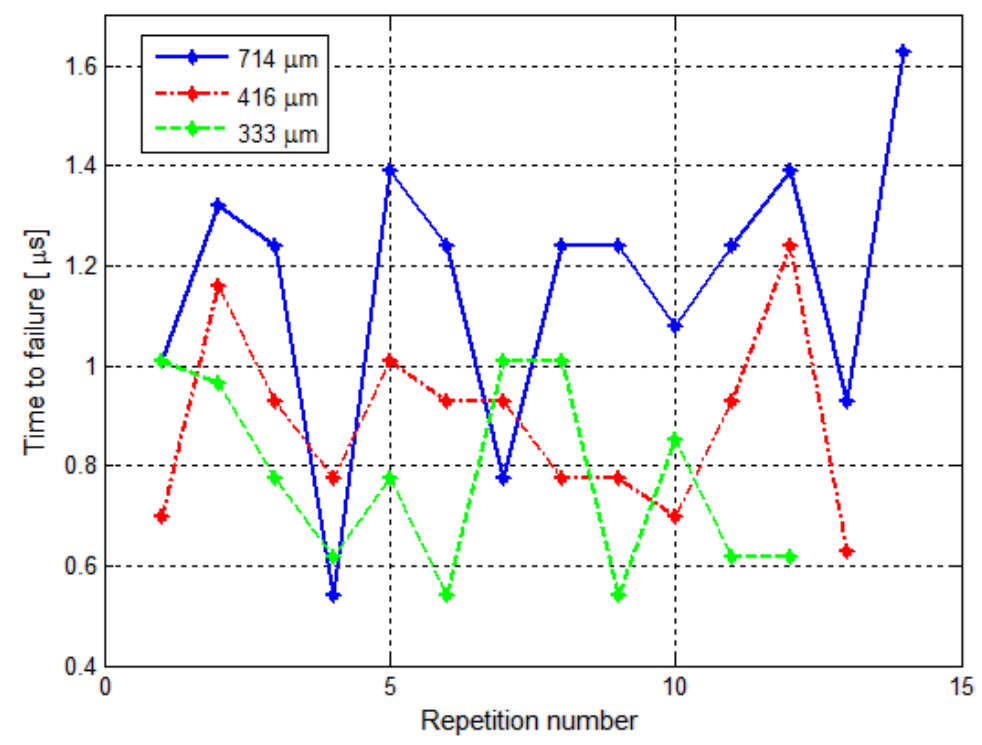

Fig. 20. Grain size effect on time-to-failure. 


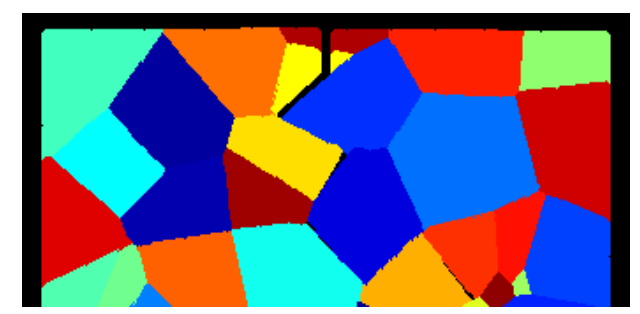

Fig. 21. Top half of the polycrystal: crack tip in proximity to the grain boundary (lowest point of the blue curve in Fig. 21).

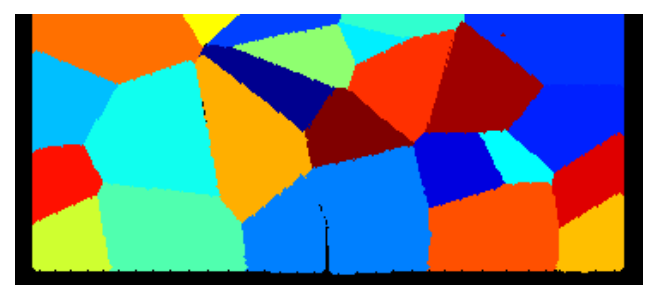

Fig. 22. Bottom half of the polycrystal: crack tip embedded inside the grain boundary (peak of the blue curve in Fig. 21).

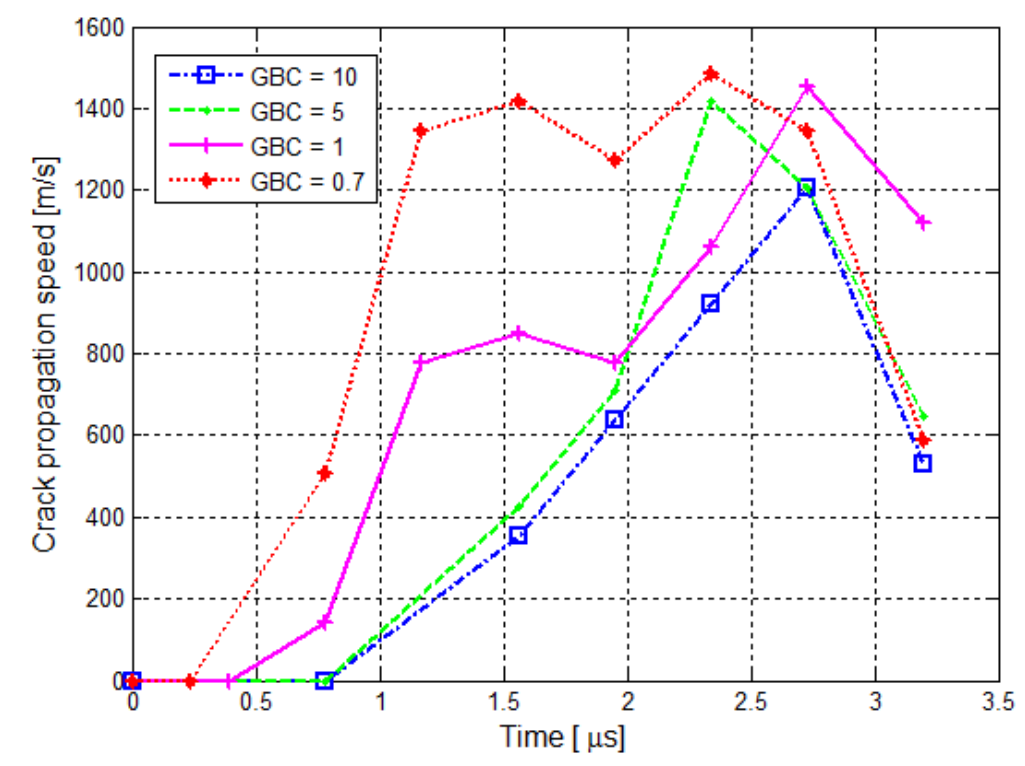

Fig. 23. Effect of GBC on crack propagation speed.
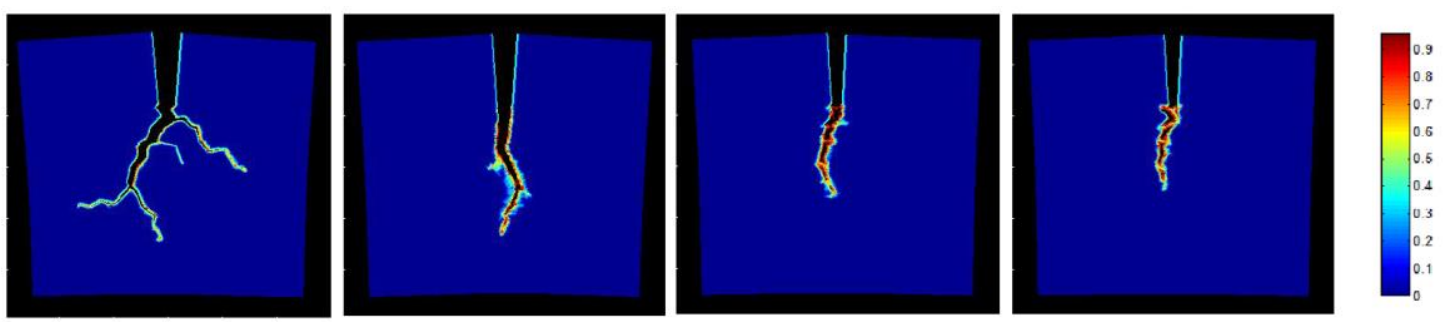

Fig. 24. Effect of GBC on damage map at time $=3.2 \mu \mathrm{s}$. From left to right: $\mathrm{GBC}=0.7, \mathrm{GBC}=1$, $\mathrm{GBC}=\mathbf{5}, \mathrm{GBC}=\mathbf{1 0}$. 

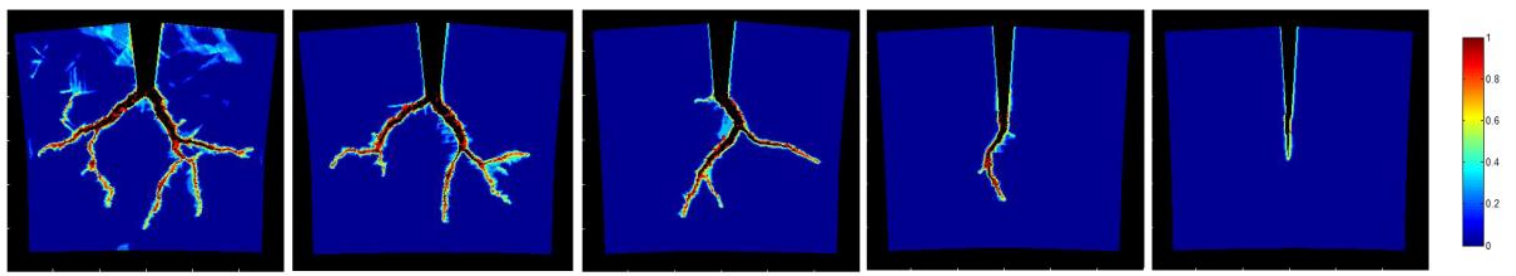

Fig. 25. Effect of $K_{I C}$ on the morphology of damage (time $=3.2 \mu \mathrm{s}$ ). From left to right:

$$
\psi=0.2, \psi=0.35, \psi=0.5, \psi=1, \psi=2 .
$$
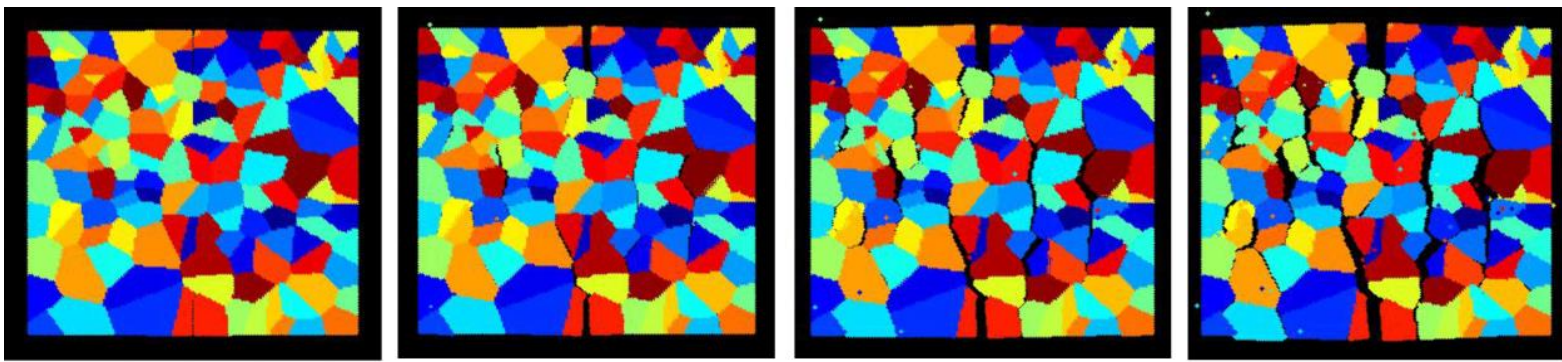

Fig. 26. Time evolution of damage in an initially-damaged polycrystal in plane stress configuration when GBC $=0.5$. From left to right: time $=1 \mu \mathrm{s}$, time $=2 \mu \mathrm{s}$, time $=3 \mu \mathrm{s}$ and time $=4 \mu \mathrm{s}$.
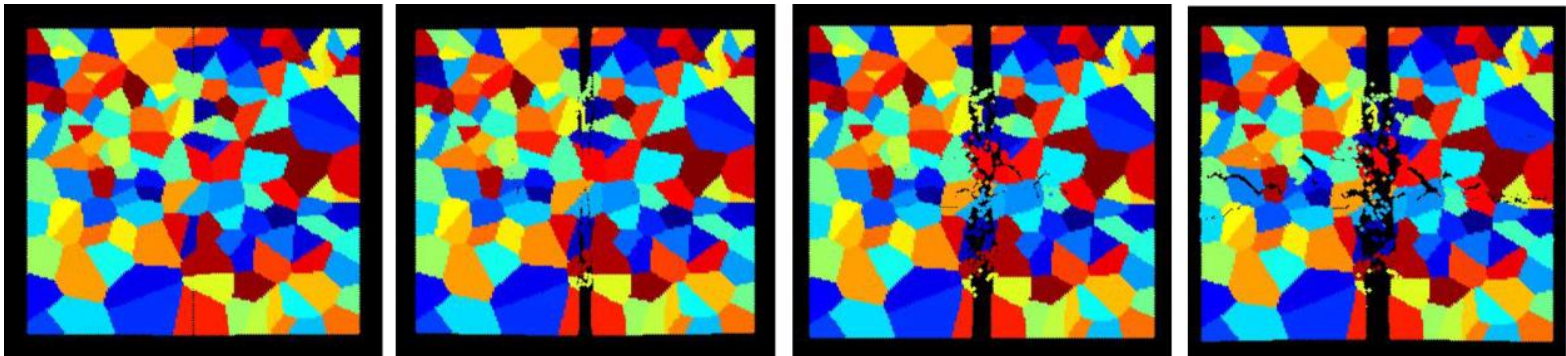

Fig. 27. Time evolution of damage in an initially-damaged polycrystal in plane stress configuration when GBC $=1.0$. From left to right: time $=1 \mu \mathrm{s}$, time $=2 \mu \mathrm{s}$, time $=3 \mu \mathrm{s}$ and time $=4 \mu \mathrm{s}$.
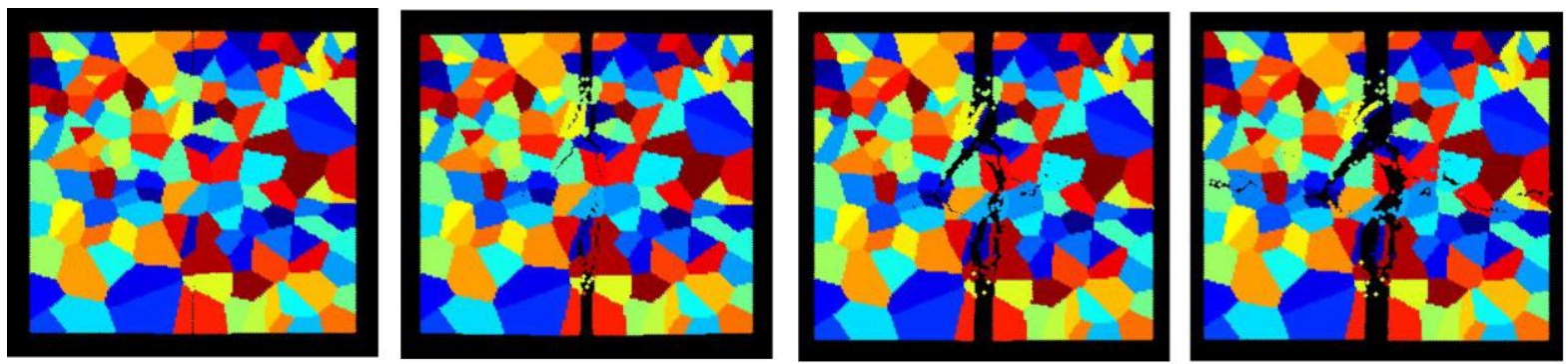

Fig. 28. Time evolution of damage in an initially-damaged polycrystal in plane stress configuration when $\mathrm{GBC}=2.0$. From left to right: time $=1 \mu \mathrm{s}$, time $=2 \mu \mathrm{s}$, time $=3 \mu \mathrm{s}$ and time $=4 \mu \mathrm{s}$. 



Fig. 29. Time evolution of damage in an initially-damaged polycrystal in plane strain configuration when GBC $=0.5$. From left to right: time $=1 \mu \mathrm{s}$, time $=2 \mu \mathrm{s}$, time $=3 \mu \mathrm{s}$ and time $=4 \mu \mathrm{s}$.
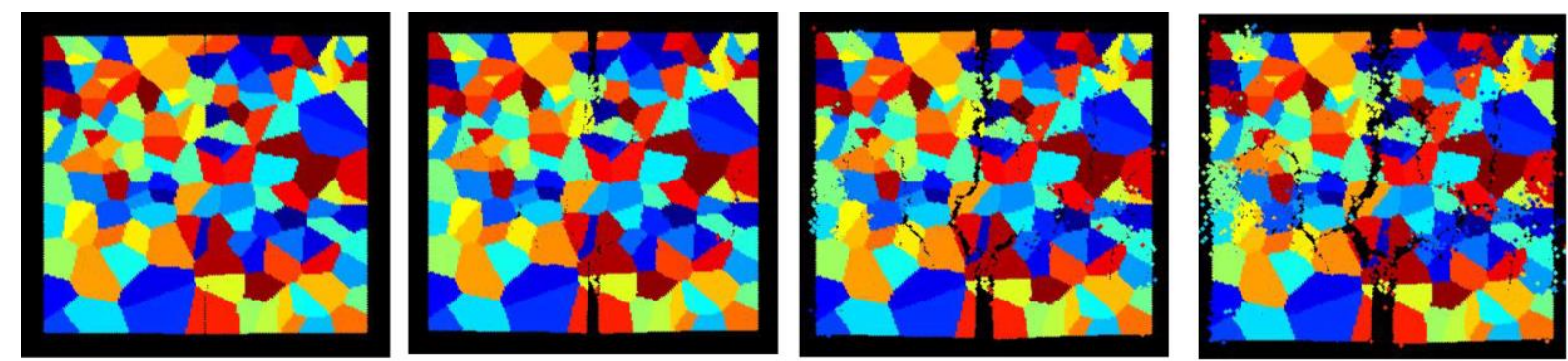

Fig. 30. Time evolution of damage in an initially-damaged polycrystal in plane strain configuration when GBC $=1.0$. From left to right: time $=1 \mu \mathrm{s}$, time $=2 \mu \mathrm{s}$, time $=3 \mu \mathrm{s}$ and time $=4 \mu \mathrm{s}$.


Fig. 31. Time evolution of damage in an initially-damaged polycrystal in plane strain configuration when $\mathrm{GBC}=2.0$. From left to right: time $=1 \mu \mathrm{s}$, time $=2 \mu \mathrm{s}$, time $=3 \mu \mathrm{s}$ and time $=4 \mu \mathrm{s}$. 


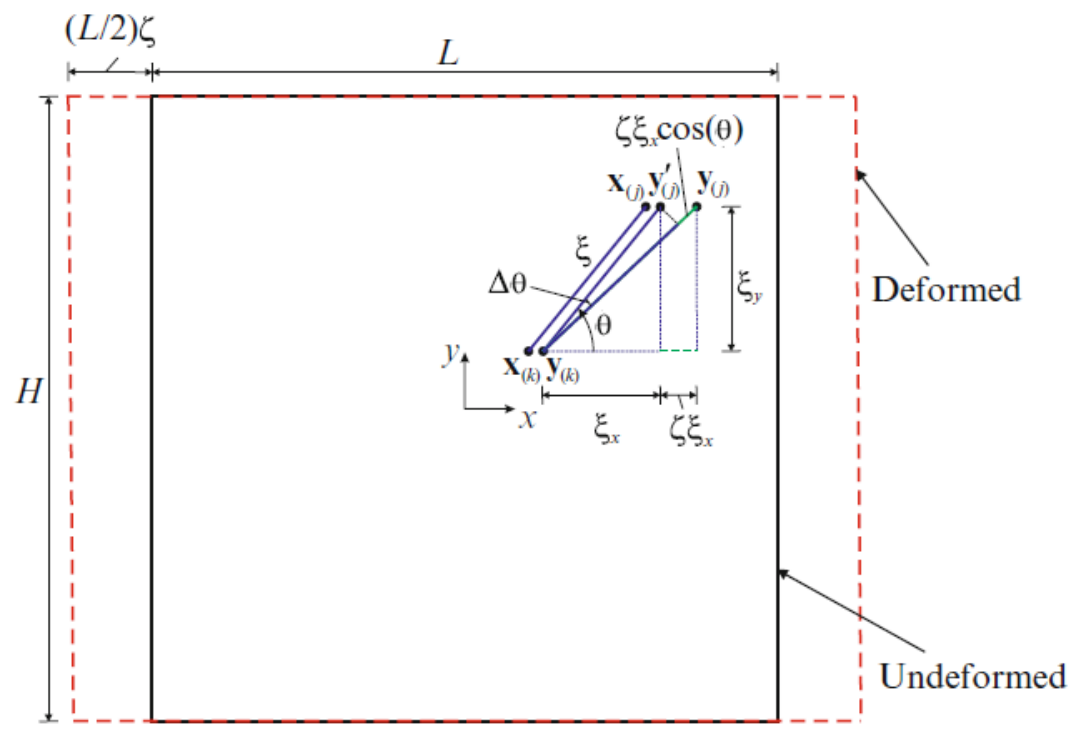

Fig. A.1. First loading condition

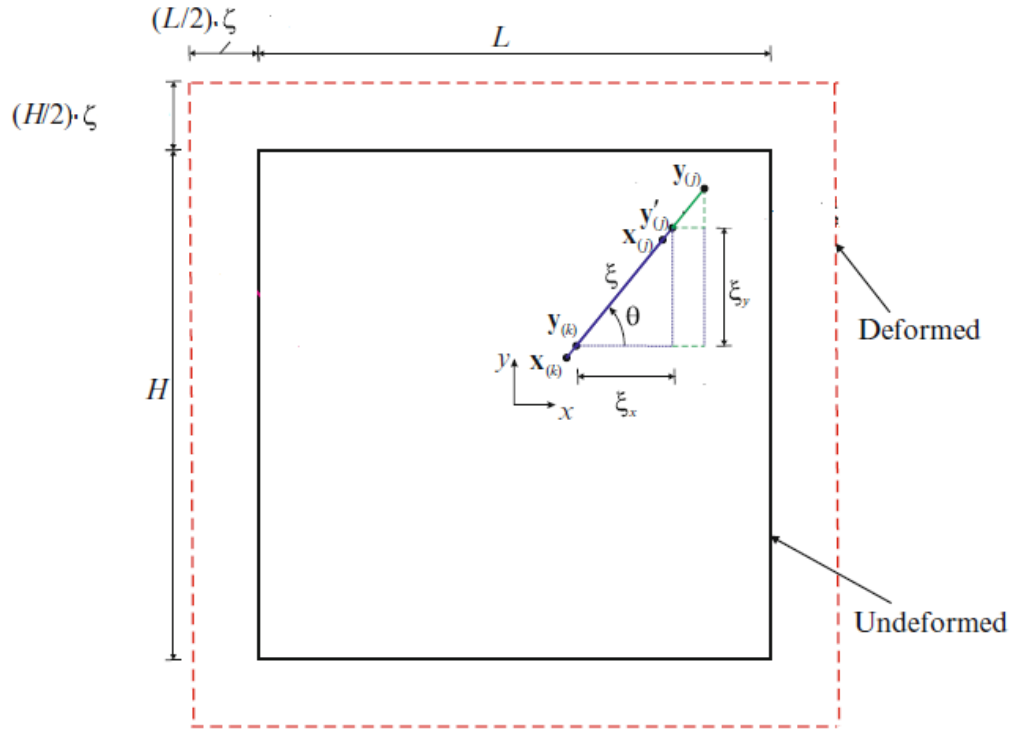

Fig. A.2. Second loading condition 


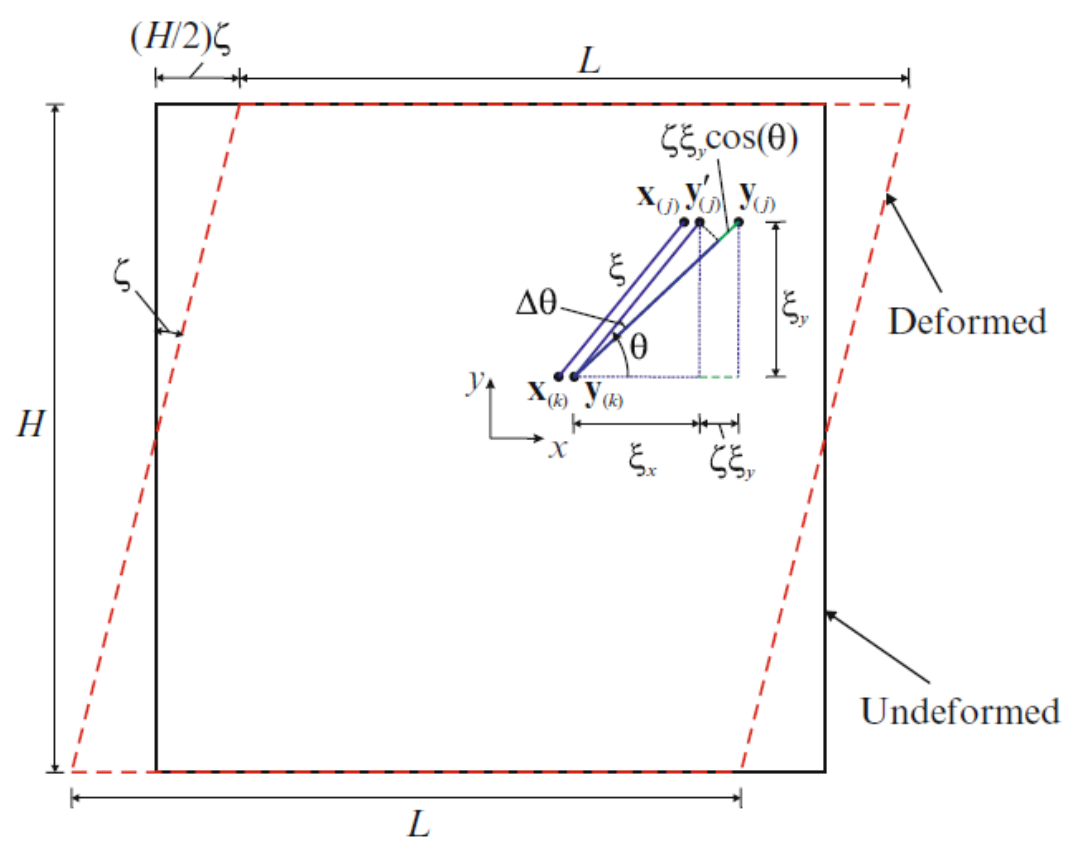

Fig. A.3. Third loading condition 
Tables

Table 1 AISI 4340 steel chemical composition

\begin{tabular}{|c|c|c|c|c|c|c|c|c|}
\hline $\mathrm{C}$ & $\mathrm{Si}$ & $\mathrm{Mn}$ & $\mathrm{P}$ & $\mathrm{S}$ & $\mathrm{Cu}$ & $\mathrm{Mo}$ & $\mathrm{Ni}$ & $\mathrm{Cr}$ \\
\hline $0.39 \%$ & $0.27 \%$ & $0.77 \%$ & $0.018 \%$ & $0.016 \%$ & $0.14 \%$ & $0.23 \%$ & $1.38 \%$ & $0.78 \%$ \\
\hline
\end{tabular}

